\title{
Der Futurismus, der Fortschritt und ein Vorläufer
}

Betrachten wir also den Beginn des von Marinetti auf den 11. Mai 1912 datierten Technischen Manifests der futuristischen Literatur, wobei dieser Einstieg - charakteristisch für die Manifeste Marinettis - erneut narrativ gewählt ist, bevor hernach eine diskursive strukturierte thesenartige Präsentationsform die Oberhand gewinnt! Zwischen den dominant narrativen und diskursiven Teilen entsteht eine vieldeutige Reibung, eine Friktion, welche der gesamten Prosa zu ihrer explosiven Wirkung verhilft:

Ich saß im Flugzeug auf dem Benzintank und wärmte meinen Bauch am Kopf des Fliegers, da fühlte ich die lächerliche Leere der alten, von Homer ererbten Syntax. Stürmisches Bedürfnis, die Worte zu befreien, sie aus dem Gefängnis des lateinischen Satzbaus zu ziehen! Dieser hat natürlich, wie alle Dummköpfe, einen vorausschauenden Kopf, einen Bauch, zwei Beine und zwei Plattfüße, aber er wird niemals zwei Flügel haben. Es reicht gerade, um zu gehen, einen Augenblick zu laufen und fast sofort wieder keuchend anzuhalten!

Das hat mir der surrende Propeller gesagt, während ich in einer Höhe von zweihundert Metern über die mächtigen Schlote von Mailand flog. Und er fügte hinzu:

1. MAN MUSS DIE SYNTAX DADURCH ZERSTÖREN, DASS MAN DIE SUBSTANTIVE AUFS GERADEWOHL ANORDNET, SO WIE SIE ENTSTEHEN.

2. MAN MUSS DAS VERB IM INFINITIV GEBRAUCHEN, damit es sich elastisch dem Substantiv anpaßt, und es nicht dem Ich des Schriftstellers unterordnen, der beobachtet oder erfindet. Nur das Verb im Infinitiv kann das Gefühl für die Fortdauer des Lebens und die Elastizität der Intuition, durch die sie wahrgenommen wird, vermitteln.

3. MAN MUSS DAS ADJEKTIV ABSCHAFFEN, damit das bloße Substantiv seine wesenhafte Färbung beibehält. Da das Adjektiv seinem Wesen nach nuancierend ist, ist es mit unserer dynamischen Vision unvereinbar, denn es setzt einen Stillstand, eine Überlegung voraus.

4. MAN MUSS DAS ADVERB ABSCHAFFEN, diese alte Schnalle, die ein Wort an das andere bindet. Das Adverb gibt dem Satz einen lästigen, einheitlichen Ton.

5. JEDES SUBSTANTIV MUSS SEIN DOPPEL HABEN, d.h. jedem Substantiv muß ohne Bindewort das Substantiv folgen, dem es durch Analogie verbunden ist. Beispiel: Mann - Torpedoboot, Frau - Meerbusen, Menge - Brandung, Platz - Trichter, Tür Wasserhahn.

Da die Fluggeschwindigkeit unsere Kenntnis der Welt vervielfacht hat, wird die Wahrnehmung durch Analogien immer natürlicher für den Menschen. Man muß folglich die Redewendungen wie, gleich, so wie, ähnlich unterdrücken. Besser noch sollte man direkt den Gegenstand mit dem von ihm heraufbeschworenen Bild verschmelzen und so das Bild mit einem einzigen, essentiellen Wort in Verkürzung wiedergeben.

6. AUCH DIE ZEICHENSETZUNG MUSS ABGESCHAFFT WERDEN. Sind Adjektive, Adverbien und Konjunktionen erst beseitigt, dann ist die Zeichensetzung natürlich aufgehoben in der wechselnden Dauer eines lebendigen, durch sich selbst geschaffenen

Ә Open Access. () 2021 Ottmar Ette, publiziert von De Gruyter. (c) BY-NC-ND Dieses Werk ist lizensiert unter einer Creative Commons Namensnennung - Nicht-kommerziell - Keine Bearbeitung 4.0 International Lizenz. https://doi.org/10.1515/9783110703450-006 
Stils, ohne die absurden Unterbrechungen durch Kommata und Punkte. Um gewisse Bewegungen hervorzuheben und ihre Richtungen anzugeben, wird man die mathematischen Zeichen: $+-\mathrm{x}:=><$ und die musikalischen Zeichen verwenden.

7. Die Schriftsteller haben sich bisher der unmittelbaren Analogie hingegeben. Sie haben zum Beispiel ein Tier mit einem Menschen oder mit einem anderen Tier verglichen, was ungefähr der Photographie gleichkommt. (Sie haben zum Beispiel einen Foxterrier mit einem ganz kleinen Vollblut verglichen. Andere, Fortgeschrittenere, könnten denselben zitternden Foxterrier mit einer kleinen Morsemaschine vergleichen. Ich hingegen vergleiche ihn mit aufkochendem Wasser. Es ist dies eine ABSTUFUNG VON IMMER AUSGEDEHNTEREN ANALOGIEN, und es bestehen immer tiefere und festere, wenn auch sehr fernliegende Beziehungen.)

Analogie ist nur die tiefe Liebe, die fernstehende, scheinbar verschiedene und feindliche Dinge verbindet. Nur durch sehr ausgedehnte Analogien kann ein orchestraler Stil, der gleichzeitig polychrom, polyphon und polymorph ist, das Leben der Materie umfassen.

Wenn ich meiner „Schlacht von Tripolis“ einen von Bajonetten strotzenden Schützengraben einem Orchester und eine Mitrailleuse einer verhängnisvollen Frau vergleiche, so habe ich intuitiv einen großen Teil des Weltalls in die kurze Episode einer afrikanischen Schlacht eingeführt.

Die Bilder sind nicht Blumen, die man mit Sparsamkeit auswählen und pflücken muß, wie Voltaire sagte. Sie bilden das Blut der Dichtung. Dichtung muß eine ununterbrochene Folge neuer Bilder sein, ohne die sie blutarm und bleichsüchtig ist [...].

8. ES GIBT KEINE BILDKATEGORIEN, die vornehm, grob oder vulgär, übertrieben oder natürlich sind [...]

9. Um die aufeinanderfolgenden Bewegungen eines Gegenstandes darzustellen, muß man die Kette der Analogien, die er hervorruft, wiedergeben, und jede Analogie muß verdichtet, in einem essentiellen Wort zusammengefaßt werden. [...]

10. Da jede Art von Ordnung notwendig das Produkt eines vorsichtigen und behutsamen Verstandes ist, muß man die Bilder orchestrieren und sie nach der GRÖSSTMÖGLICHEN UNORDNUNG verteilen.

11. MAN MUSS DAS „ICH“ IN DER LITERATUR ZERSTÖREN, das heißt die ganze Psychologie. [...]

Außerdem müssen drei Elemente in die Literatur eingeführt werden, die bisher vernachlässigt wurden:

1. DER LÄRM (Manifestation des Dynamismus der Gegenstände);

2. DAS GEWICHT (Flugvermögen der Gegenstände);

3. DER GERUCH (Streuvermögen der Gegenstände). [...]

Zusammen werden wir erfinden, was ich DRAHTLOSE PHANTASIE nenne. ${ }^{1}$

Bei diesem hier ungewöhnlich lange, wenn auch in Auszügen zitierten Manifest wird zunächst einmal der narrative Einstieg und ein Ort des Sprechens skizziert,

1 Marinetti, Filippo Tommaso: Technisches Manifest der futuristischen Literatur. In: Asholt, Wolfgang / Fähnders, Walter (Hg.): Manifeste und Proklamationen, S. 24-26. 
der nicht nur überaus originell, sondern auch im Kontext unserer Fragestellungen signifikant und epistemologisch relevant ist. Denn der Ich-Erzähler sitzt auf dem Benzintank in einem Flugzeug, mit dem er gerade die mächtigen Schlote der Fabrikstadt Mailand überfliegt. Es ist daher eine Industrielandschaft in Italiens hochentwickeltem Norden, welche aus der Vogelperspektive wahrgenommen wird. Und es ist dabei selbstverständlich, dass der Ich-Erzähler gleichsam auf einem Pulverfass sitzt, das in seinem fliegenden technischen Apparat jederzeit explodieren kann, zugleich aber auch als Benzintank für jene Energie und jenen Treibstoff steht, die alles vorantreiben.

Von dieser mobilen und erhöhten Position aus - es ist gleichsam die erhabene Position einer modernisierten Ästhetik - wird eine Überflieger-Perspektive eingenommen, von der aus die literarische Tradition sich als jene Homers erweist, die bis vor kurzem noch im Schwange gewesen sei. Doch was kann eine solche, selbstredend überkommene Syntax angesichts der aktuellen Wahrnehmung unserer Welt den heutigen Menschen noch sagen? Die herkömmliche literarische Tradition und deren Syntax erscheinen uns so als etwas angesichts des technischen Fortschritts und der veränderten Bedingungen der Kunstproduktion völlig Leeres und Überholtes. Sie können nicht länger Schritt halten mit dem Fortschritt und einem Futur, das im Zeichen schneller Bewegungen steht, welchen sie mit ihren Plattfüßen nur mehr hinterher zu hecheln vermögen.

Es existiert also eine Inkongruenz zwischen der Position der Moderne und der Geschwindigkeit des Neuen einerseits und der Behäbigkeit und Leere einer veralteten, dem Ursprung der abendländischen Literatur entsprungenen Syntax andererseits, die dieser aktuellen Welt nicht mehr gerecht $\mathrm{zu}$ werden versteht. Homer? Das war gestern! Die Glieder seines geordneten Satzbaues? Die gilt es zu zerstreuen und zu zerlegen, ja zu zerstören!

Die Position des Ichs ist also die einer beschleunigten Moderne, für die das Flugzeug und der an Bord befindliche Dichter höchstselbst stehen, vor deren Geschwindigkeit aber die bisherige Literatur gleichsam einen Quantensprung machen muss. Aus alledem ergibt sich der heroische Gestus einer Befreiung der Worte, einer Befreiung, die im Übrigen auch zu den „parole in libertà“ führen wird, jenen Worten in Freiheit, zu einer Radikalisierung des vom Lyriker Marinetti zunächst benutzten „vers libre“ und letztlich zu für die Lyrik des 20. Jahrhunderts insgesamt bahnbrechenden Umwälzungen. Der Futurismus hat diese Entwicklungen bei weitem nicht alle erdacht und implementiert, aber er spielte eine wichtige Rolle dabei, sie konkret auf den Weg zu bringen und zu Beginn des 20. Jahrhunderts eine Umwertung aller Werte voranzutreiben.

Die Inkongruenz zwischen technologischem Fortschritt und literarischer Sprache, Transformation der Gesellschaft und Stillstand von Kunst und Literatur, zwischen veränderter Wahrnehmung der Welt und gleichbleibender literarisch- 
künstlerischem Ausdruck impliziert in Marinettis Technischem Manifest der futuristischen Literatur mithin die Legitimation einer Befreiungstat, eben jener des Ich-Erzählers. Eine Ästhetik des Bruchs zieht nun auch für die Sprache der Literatur herauf: Das Gefängnis des lateinischen Satzbaus müsse zerstört werden, womit Homer und das Griechentum nun um Rom und die lateinischen Traditionen der Antike erweitert werden, die noch immer alles beherrschten. Mit dieser totalen Dominanz müsse nun aber endgültig Schluss gemacht werden! Hübsch ist, dass dieser lateinische Satzbau mit etwas Organischem, Figürlichem in Beziehung gebracht wird: einem menschlichen Körper mit zwei Armen und Plattfüßen, denen aber ausgerechnet zwei Flügel fehlen, also just jene technischen Artefakte, deren sich der Ich-Erzähler in seinem Flugzeug sitzend selbst bedient. Damit wird jegliche schlichte Reparatur des lateinischen Satzbaus und Versbaus über Bord geworfen: Etwas völlig Neues müsse her!

Vor diesem Hintergrund ist es alles andere als überraschend, dass es ein technisches Artefakt ist, der surrende Propeller des Flugzeugs nämlich, der dem Ich das ebenfalls technische Manifest einflüstert, das wir glücklicherweise in Buchstabenschrift lesen können. Alle Vorschläge und Proklamationen sind wie stets bei Marinetti fein säuberlich und buchhalterisch, aber natürlich auch recht effektiv in einzelne Punkte aufgeteilt. Aus didaktischen Gründen ist dabei das Schriftbild verändert, eine nicht unwichtige Tatsache, welche in diesem Manifest freilich noch ganz traditionell die zentralen Forderungen des Futurismus zu unterstreichen bemüht ist.

Nicht ganz unbedeutend erscheint mir aber auch, dass es gerade der Propeller der Flugmaschine ist, welcher zum Ich-Erzähler spricht. Denn dieser Propeller ist nicht nur ein Artefakt, das die Bewegung des Motors in Geschwindigkeit und Vorwärtsbewegung umsetzt. Er ist zugleich Vorbild für die Entwicklung jenes anderen Propellers, der mit dem Kinematographen just jene Bewegung zauberte, die sich aus hintereinander geschalteten Einzelbildabläufen zusammensetzt. Sie wissen ja: Die statischen Bilder werden nur kinotechnisch mobilisiert durch die ständige, unseren Augen nicht mehr bewusste Unterbrechung der Bildprojektion durch eine durchlaufende Flügelschraube, die ganz dem Propeller des Flugzeugs verwandt ist. Mit Hilfe dieser Flügelschraube oder dieses Propellers haben die Bilder laufen gelernt.

In diesem Zusammenhang ist es ebenso wenig zufällig, dass es genau dieses ,Durchschießen' von Lichtstrahlen durch den Propeller ist, was auch das Durchschießen von Maschinengewehrsalven durch den Propeller eines Militärflugzeugs erlaubt. Technisch gesprochen ist das absolut identisch! Ich möchte an dieser Stelle auf die grundlegende Studie von Friedrich Kittler verweisen, die unter dem Titel Grammophon Film Typewriter als eine der umstrittensten Habilitationsschriften in die Geschichte der Freiburger wie der deutschen Habilitationen ein- 
gegangen ist. Sie erschien 1986 in Buchform ${ }^{2}$ und belegte auf eindrückliche Weise, dass grundlegende Neuansätze mit allerlei Brüchen innerhalb herkömmlicher wissenschaftlicher Disziplinen einherzugehen pflegen. Glücklicherweise gelang es nicht, Friedrich Kittler und seine Schrift zu disziplinieren! Kittler arbeitete auf kulturwissenschaftlich-medienanalytische Weise die grundlegenden Zusammenhänge zwischen den neuen Medien Typewriter oder Schreibmaschine, Film oder Kinematograph und Grammophon mit all jenen grundlegenden Technologien heraus, welche für die Entwicklung moderner und modernster Waffen im Übergang zum 20. Jahrhundert maßgeblich waren. Dieser Band, der in Duktus und Habitus durchaus aufhorchen ließ, verweist uns zusätzlich auf die enorme Wichtigkeit des Krieges für die Fortschritte und Entwicklungen gerade auch in Kunst und Literatur. Auch wenn man nicht der These zustimmen muss, der zufolge der Krieg der Vater aller Dinge sei, so bietet dieser Band doch mancherlei Einsichten, auf die ich im Folgenden auch zurückgreifen möchte.

Ohne hier auf die technische Problematik des Kinematographen vertieft eingehen zu können, will ich an dieser Stelle doch auf die Illusion der Bewegung hinweisen, wie sie gegen Ende des 19. Jahrhunderts auf verschiedenste Weise erzeugt wurde. Führen wir uns dabei kurz einmal das sogenannte Daumenkino oder Abblätterbuch vor Augen, das ebenfalls durch das Vorbeirauschen der Seiten jeweils unterbrochen durch die durchlaufende Seite der Buchseiten - unserem Auge Bewegung vorgaukelt: Wie Statik scheinbar in Dynamik und Bewegung umgewandelt werden kann, zeigt dieses Ihnen allen sicherlich vertraute Beispiel recht deutlich.

Weiter möchte ich Ihnen nur kurz die oftmals diskutierte Problematik vorführen, die sich für die Zeitgenossen des 19. Jahrhunderts mit der Frage verband, ob ein galoppierendes Pferd tatsächlich - wie es in der Malerei oftmals dargestellt wurde, zu einem bestimmten Zeitpunkt keines seiner Hufe auf dem Boden hat; oder - wie es einmal ein ungeschickter Kollege formulierte, der sich damit dem Lachen seiner Zuhörer preisgab - ob ein Pferd (oder auch ein Dackel) tatsächlich alle vier Beine unter dem Bauch hat. Die Frage wurde spätestens durch die seriellen Aufnahmen von Muybridge in der zweiten Hälfte der 1880er Jahre beantwortet: Vereinfacht gesagt, waren die Konventionen der europäischen Malerei schlichtweg falsch. Die sehr unterschiedliche Darstellung des Pferdes, des galoppierenden Pferdes und von Bewegung überhaupt mag nach Ende des 19. Jahrhunderts ein Licht darauf werfen, wie der technische Fortschritt die Darstellungsmodi der Kunst auf Grund veränderter Wahrnehmungsbedingungen buchstäblich revolutionierte.

2 Kittler, Friedrich: Grammophon Film Typewriter. Berlin: Brinkmann \& Bose 1986. 


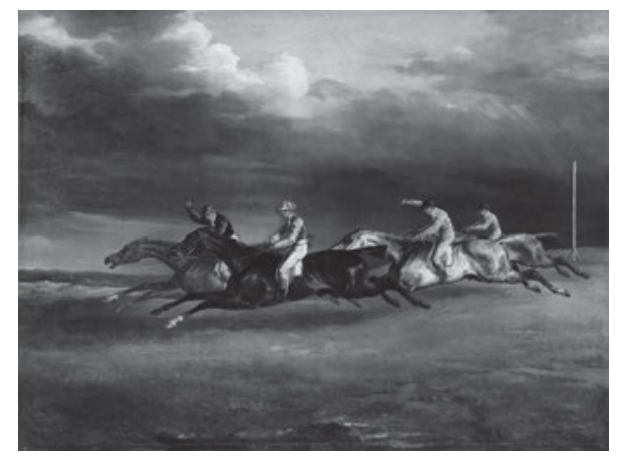

Abb. 28: Théodore Géricault: Das Derby in Epsom, Öl auf Leinwand, 1821.

Hohe Geschwindigkeiten wurden nun nicht nur anders wahrgenommen, sie wurden auch anders speicherbar: Literatur und Malerei verloren auf diesem Gebiet ihre Monopolstellungen. Damit veränderten sich fast schlagartig unsere Wahrnehmungsgewohnheiten - und selbstverständlich auch deren künstlerischliterarische Darstellungsweisen. Muybridge gelang der photographische Beweis, dass es Abschnitte im Bewegungsablauf von Pferden gibt, zu denen in der Tat kein einziges der vielen Hufe auf dem Boden ist. Dieser für die Zeitgenossen zunächst verblüffende Bewegungsablauf sah gänzlich anders aus, als sich die Zeitgenossen, die doch viel Erfahrung mit Pferden hatten, dies gewöhnlich vorstellten. Ich darf Ihnen dies bewegungsphotographisch anschaulich vor Augen führen! ${ }^{3}$ Diese Bilder finden Sie in allen klassischen Darstellungen zur Geschichte von Photographie, Kinematograph und Bewegung:

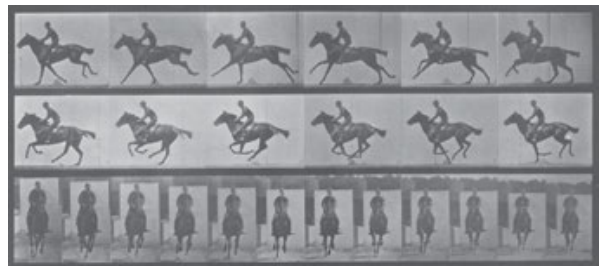

Abb. 29: Eadweard Muybridge: Horse and Rider Galloping, Chronofotografie, 1883-1887.

Wir sind noch nicht einmal richtig bis zur ersten These von Marinettis Technischem Manifest vorgedrungen. Doch sollte Ihnen unsere Beschäftigung mit der Aufnahme und künstlerischen Potenzierung aller Aspekte rund um Bewegung zeigen, dass sich aus all diesen Fragestellungen technisch-künstlerische Apparaturen entwickeln ließen, die unsere heutige Wahrnehmung ganz selbst-

3 Vgl. Chronophotographie, Muybridge 1887; Chronophotographie, Muybridge 1884-1887; sowie Cinematograph und Dreiflügelblende. 
verständlich prägen. Sie sind uns also buchstäblich in Fleisch und Blut übergegangen ...

Aus dem Zerschneiden bestimmter durchgängiger, kontinuierlicher Bewegungsabläufe ergibt sich geradezu zwangsläufig die Technik der künstlerischen Schnitte oder der Montage, die ja gerade auf dem Prinzip des Diskontinuierlichen, also des gegen das Kontinuierliche Gerichteten, beruht. Sie verstehen nun besser, warum bei Marinetti die Bewegung des lateinischen Satzes die eines mehr oder minder gemächlichen Gehens sein musste und nicht etwa die einer sprunghaften Fortbewegung. Denn jetzt ist eine neue künstlerische Erfahrung hinzugetreten, die sich als ungeheuer fruchtbar erweisen sollte. Dass übrigens auch die Chronophotographie eine direkte Beziehung zum militärischen Bereich, insbesondere zum Schießgewehr, hat, mag Ihnen eine Abbildung aus Friedrich Kittlers Band zeigen, die Ihnen Mareys damals berühmte chronophotographische Flinte vor Augen führt. ${ }^{4}$ Sie kann uns daran erinnern, dass auch wir in unserem Alltagsleben noch ,Bilder schießen':

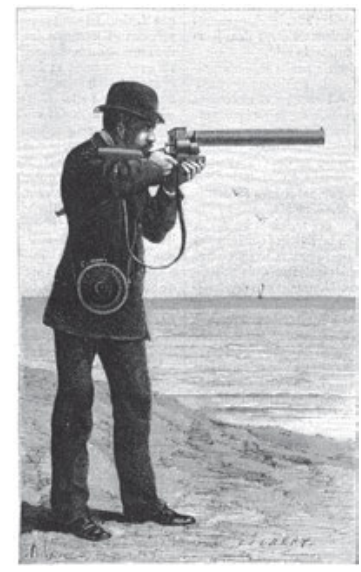

Abb. 30: Étienne-Jules Marey: Chronophotographische Flinte, 1882.

Bleiben wir noch einen Augenblick scheinbar abseits von Marinettis Technischem Manifest, mit dem wir uns gleich wieder näher auseinander setzen wollen, und beschäftigen wir mit jenen Fragen, die uns die technischen Medien der Zeit als solche stellen! In seinem schönen, wenn auch bisweilen etwas selbstverliebten Buch hat Friedrich Adolf Kittler zurecht darauf aufmerksam gemacht, dass in der zweiten Hälfte des 19. Jahrhunderts bestimmte Sinneswahrnehmungen zum ersten Male speicherbar gemacht werden konnten. Dazu gehört auch der Bereich

4 Vgl. Kittler, Friedrich: Grammophon Film Typewriter, S. 189. 
des Hörens: 1877 bereits hatte Edison seinen Phonographen stolz der Welt präsentiert. Seit dieser Epochenschwelle gibt es Speichermedien, die akustische und optische Daten in ihrem Zeitfluss selber festhalten und wiedergeben können. ${ }^{5}$ Die ethnologischen Museen Berlins und damit auch das aktuelle Humboldt-Forum besitzen eine Vielzahl von alten Tonwalzen, auf denen etwa die Gesänge amerikanischer oder ozeanischer indigener Gruppen aufgenommen und für die Nachwelt aufbewahrt wurden - eine nicht nur individuell spannende, sondern für die Forschung kostbare Quelle für weitergehende Untersuchungen, die uns das ,Bild“ einer weitgehend verlorenen Klanglandschaft zu geben vermögen. Dieses neue Speichermedium wurde also rasch gerade auch für wissenschaftliche Zwecke eingesetzt.

Aber auch die Entwicklungen im Bereich der Kunst waren immens und folgenreich: Denken Sie nur etwa an die Entwicklung der Filmindustrie, der eigentlichen kulturellen Schwerindustrie, im Vergleich $\mathrm{zu}$ der sich die Bereiche der Literatur mit ihren Verlagen und Akademien bestenfalls als eine Leichtindustrie bezeichnen lassen! Es war ein entscheidender Durchbruch, die unterschiedlichsten Wahrnehmungsbereiche des Menschen auf sinnlich nachvollziehbare Weise nunmehr speichern und verfügbar halten zu können. Dass wir dies mittlerweile nicht mehr nur analog, sondern vollständig digitalisiert können, ist aus dieser Perspektive zwar ein gewaltiger, aber eigentlich kein so beeindruckender Fortschritt. Gewiss vermögen wir nun Schrift, Bild, Film, Ton und viele Kombinatoriken digital ineinander überführen; aber entscheidend war es doch, überhaupt erst die Speichermöglichkeiten für das Hören, für Bewegung und eine automatisierte Buchstabenschrift gefunden zu haben! Damit möchte ich die wunderbaren Möglichkeiten einer vernetzten Digitalisierung nicht kleinreden, aber doch Ihr Bewusstsein dafür schärfen, dass am Ausgang des 19. und zu Beginn des 20. Jahrhunderts entscheidende mediale Veränderungen und Fortschritte eintraten, welche auf allen Gebieten von Kunst und Literatur enorme Folgen zeitigten. Dies in die Entfaltungen der literarischen Geschichte im 20. Jahrhundert nicht miteinzubauen, wäre eine sträfliche Reduktion!

Aber halten wir fest: Nicht umsonst haben Phonograph und Kinematograph sowie die zuvor erfundene Licht-Schrift der Photographie in ihren Bezeichnungen das ,Schreiben“ im Zentrum. Denn was sie speicherten, war letztlich die Zeit. Es ging folglich um ein Schreiben, das im Grunde eine Kunst in der Zeit ist, im Gegensatz zur Malerei, welche eine Kunst im Raum ist. Gewiss ist dies angesichts aller medialen Übergänge etwas vereinfacht ausgedrückt; aber Sie sollen sich diesen

5 Vgl. Ebda., S. 10. 
grundlegenden Gegensatz zwischen einer Zeit-Kunst (der Literatur) und einer Raum-Kunst (der Malerei) plastisch vorstellen können.

Durch Speicherung der Zeit wird diese künstlerisch in anderer Weise - und nicht mehr nur literarisch - verfügbar gemacht. Was die Literatur mit ihren sechsundzwanzig Buchstaben aneinanderreiht, kann nun ebenso gespeichert werden wie das, vor dem traditionellerweise die Literatur kapitulieren musste: das Geräusch, das nicht mehr re-alphabetisiert werden konnte. Damit taten sich nun ganze Medienwelten oder, anders ausgedrückt, menschliche Wahrnehmungswelten auf. Friedrich Adolf Kittlers These nun lief darauf hinaus, dass mit Thomas Alva Edisons großen Erfindungen, dem Kinematographen und dem Phonographen, unsere Gegenwart beginnt und wir zur Vervollständigung noch ein drittes Element aufnehmen mussten. Dieses dritte Element war nichts anderes als die Schreibmaschine, die - mittlerweile ebenfalls in digitalisierter Form - für unsere alltägliche Gegenwart unentbehrlich geworden ist. Das aber ist so ganz selbstverständlich nicht - und schon gar nicht folgenlos ...

Bis 1865 ist die Schrift, wie Friedrich A. Kittler formulierte, ${ }^{6}$ eine Tinten- oder Bleistiftspur eines Körpers auf einer Schreibunterlage. Fortan aber nutzte dieser Körper nicht mehr nur seitens der Distribution von Schrift die Maschine (also auf Ebene der Druckerpresse, der zentralen Erfindung der Gutenberg-Galaxis), sondern nun auch auf Ebene der Produktion in Gestalt der Schreibmaschine. Auch hier möchte ich Ihnen zur Verdeutlichung eine Abbildung mit auf den Weg geben, nämlich die berühmte Schreibkugel Friedrich Nietzsches. Es war wohl kein Zufall, dass es ausgerechnet dieser Philosoph war, der wohl als erster Philosoph eine Art Schreibmaschine benutzte. Aber er verwendete sie nicht nur, er kämpfte förmlich mit ihr und beobachtete sie sehr tiefsinnig in ihren medialen und nicht zuletzt künstlerischen sowie gedanklichen Auswirkungen. Denn wie er in einem Brief einmal formulierte: „Unser Schreibzeug arbeitet mit an unseren Gedanken.“7

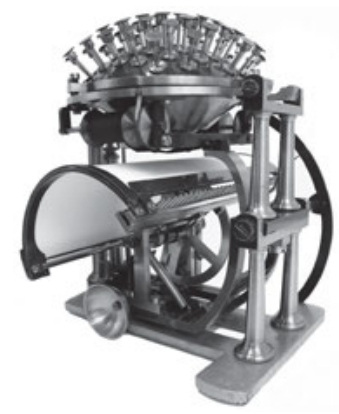

Abb. 31: Friedrich Nietzsches restaurierte Schreibkugel.

6 Ebda., S. 25.

7 Zitiert nach ebda., S. 288/9. Dort auch das Bild der Schreibkugel Nietzsches. 
Bitte bedenken Sie dabei, dass dies grundsätzliche Veränderungen für den Bereich des Zusammenhanges zwischen Körper und Schrift mit sich bringt: Denn in unserer Handschrift drückt sich unser gesamter Körper aus. Wir geben jedem Buchstaben eine spezifische Gestalt, legen einen persönlichen Druck in das Schreiben eines jeden einzelnen, wobei Form und Intensität jeder persönlichen Handschrift hochgradig individualisiert sind. Der Handschrift können wir darüber hinaus Stimmungen entnehmen, in denen wir uns gerade befinden, von der tiefen Depression bis zum euphorischen Höhenflug unseres Geistes oder zumindest unserer Laune. All dies verschwindet aber, wenn in der Beziehung zwischen Körper und Schrift eine Maschine ins Spiel kommt.

Denn mit der Schreibmaschine verblasst der individuelle Körper zum größten Teil, der in der Handschrift noch vollständig erkennbar war. Ich sage zum größten Teil, weil in der Tat Restbestände bleiben: Jede Schreiberin und jeder Schreiber drückt bei einer Schreibmaschine auf andere Weise etwa auf den in der deutschen Sprache häufigsten Buchstaben, das „Е“. Hier lassen sich deutliche Unterschiede festmachen, so dass es früher Spezialisten dafür gab, einen bestimmten Schreibmaschinentypus mit einer körperlichen Individualität kriminalistisch $\mathrm{zu}$ erfassen und $\mathrm{zu}$ analysieren. Und deshalb schnitten gewitzte Verbrecher lieber fertige Buchstaben aus einer Zeitung aus, um ihre Drohbriefe auszuformulieren. Der Körper war also in der Schreibmaschine noch nicht gänzlich verschwunden.

Eben dies aber ist beim Computer der Fall: Es ist unverkennbar, dass beim Computer der Körper des Schreibenden oder der Schreibenden vollständig ausgeschaltet wird - sicherlich zur Freude der Korrektoren von Referaten und Hausarbeiten etwa, die sich nicht länger mit unterschiedlichsten Entzifferungen aufhalten müssen. Zum einen ist folglich der individuelle Körper aus der Computertastatur verschwunden, da es keinen Unterschied macht, ob wir eine Taste nur leicht berühren oder unseren vollen Fingerdruck darauf abladen. Zum anderen reihen sich Sätze und Worte nunmehr in Buchstabenreihen an, die förmlich dazu einladen, diese Ketten zu unterbrechen, anders zu reihen, völlig neu zu ordnen, zu zerstückeln und wieder neu zusammenzusetzen. Glauben Sie es einem Menschen, der in seiner akademischen Laufbahn noch mit Handschrift und Schreibmaschine begann, dann auf elektrische Schreibmaschinen umstieg, danach auf eine komfortable Speicherschreibmaschine, bevor schließlich die ersten noch nicht IBM-tauglichen Computergenerationen und endlich die PCs, die Personal Computer, auftauchten - mitten in der Niederschrift meiner Dissertation, die ich noch auf einer Speicherschreibmaschine begann, um sie dann auf einem Computer fertig zu schreiben! Es ist ein völlig anderes Schreiben, wenn man ständig die Buchstabenreihungen anders anordnen kann, so wie ich es jetzt zum Beispiel tue ... 
Sie merken vielleicht schon, worauf ich an dieser Stelle hinauswill: auf jene dadaistische und später surrealistische Methode, Buchstaben auseinanderzuschneiden und später nach bestimmten Prinzipien - etwa dem des Zufalls wieder aneinanderzufügen. Die Montage war im Grunde eine Art Vorwegnahme jener Möglichkeiten, die wir heute auf Ebene unserer Desktops, Notebooks oder iPads ganz selbstverständlich haben - allerdings, um mit Marinetti zu sprechen, noch immer in der Syntax Homers. Zu dieser künstlerischen Methode, die erstmals Tristan Tzara, der große Dadaist, in Umlauf gesetzt hat, möchte ich aber erst später Stellung nehmen, sobald wir uns mit dem Dadaismus und den nachfolgenden historischen Avantgarden beschäftigen.

Halten wir aber an dieser Stelle mit Friedrich A. Kittler fest, dass mit der technischen Ausdifferenzierung von Optik, Akustik und Schrift, wie sie um 1880 Gutenbergs Speichermonopol sprengte, der sogenannte Mensch machbar geworden war. ${ }^{8}$ Sein Wesen, folgen wir Kittler, läuft über zu Apparaturen, Maschinen übernehmen nun Funktionen des Zentralnervensystems und nicht mehr bloß, wie noch zuvor, die schiere Muskulatur. Dies bedeutet, dass in der Konsequenz erstens der Entwicklung all dieser Speichermedien und zweitens im Verbund mit den Möglichkeiten vernetzter Digitalisierung eine Künstliche Intelligenz (KI) entwickelt werden kann, die im Verlauf der Jahrzehnte nach Erscheinen von Kittlers Studie weitreichende Formen angenommen hat. Dies bedeutet wiederum ganz ohne Zweifel, dass futuristische Ideen und Vorstellungen zum Teil längst in Alltagserfahrungen übergegangen sind! Zugleich enthalten viele der künstlerischen Zielsetzungen in den futuristischen Manifesten - jenseits der verabscheuenswürdigen Verstrickungen mit faschistischer Ideologie - noch immer eine Vielzahl an wegweisenden Herausforderungen, die auch für unsere Zeit künstlerisch produktiv sind.

Wir stehen in diesem historischen Kontext am Beginn der Erschaffung künstlicher Intelligenz und zugleich vor jener Trennung zwischen Materie und Information, welche unsere heutige Gegenwart noch immer prägt. Natürlich ist es ebenso aufschlussreich wie zukunftsweisend, dass die von Friedrich Kittler erläuterten drei Ebenen in unserer Zeit, ein gutes Jahrhundert später, zusammengeführt und miteinander verschaltet werden können. Aktuelle Entwicklungen paraphrasierend ließe sich sagen: Die Compact Disc (CD) digitalisiert das Grammophon, die Digitalkamera ebenso Kino wie Video. Alle Datenströme münden in Zustände von Turings Universaler Maschine, Zahlen und Figuren werden (der Romantik zum Trotz) Schlüssel aller Kreaturen. Dies ist, kurz zusammengefasst, der jetzige

8 Vgl. Kittler, Friedrich: Grammophon Film Typewriter, S. 29. 
Zustand, an den sich noch immer manche der futuristischen Bestrebungen und Zielsetzungen anschließen ließen.

Wir wollten uns mit diesem kurzen Exkurs einige Grundlagen dafür erarbeiten, dass sich ebenso die Wahrnehmung und die Wahrnehmungsweisen wie die Darstellung und die Darstellungsweisen innerhalb dieser künstlerischen und literarhistorischen Entwicklung neu konfigurierten. Auf diese Weise wurden Techniken wie Montage und Collage, ${ }^{9}$ beispielsweise im Kubismus oder in der avantgardistischen Literatur, geradezu selbstverständlich, wie dies etwa die Präsentation der Objekte im Kubismus zeigt: Gegenstände, die wir aus mehreren Blickwinkeln zugleich erfassen und die uns daher ein polylogisches, mehreren Logiken und Perspektiven gleichermaßen verpflichtetes Bild gewähren. Schnitte, Montagen und Collagen wurden aber auch zur Grundlage einer neuen Kunst, nämlich der kinematographischen Kunst der Bilderkombination, die uns in Fleisch und Blut übergegangen ist. Und dennoch gibt es auch hier schon längst wieder generationelle Unterschiede: Ihre Generation etwa ,verträgt“ und verarbeitet eine wesentlich höhere Geschwindigkeit und Anzahl an Schnitten als die meinige, die noch etwas näher dran ist an der Syntax Homers und der damit verbundenen scheinbaren Gemächlichkeit.

Kehren wir nun an dieser Stelle, mit zahlreichen Einblicken in grundlegende technische Veränderungen und Transformationen ausgestattet, zu Filippo Tommaso Marinettis Manifest zurück! Dieses wurde ja - wie wir wissen - aus der Perspektive eines Ich-Erzählers verfasst, der an den Benzintank seiner fliegenden Maschine gepresst ist und dem der Propeller eines Flugzeugs diktierte, was die künftige Entwicklung der Kunst sein werde oder zu sein habe. Sehen wir uns jetzt erst einmal an, was er dem avantgardistischen Künstler alles geflüstert hat!

Die erste Regel, ganz in Großbruchstaben aufbereitet, besagt, dass die Syntax zerstört werden soll, und zwar dadurch, dass man die Substantive nun aufeinander folgen lässt aufs Geratewohl, so dass ein Zufallsprinzip hier ebenso eingeführt wird wie die Kontingenz der entsprechenden Abfolge im Satzgefüge. Dies bedeutet letztlich immer auch die Möglichkeit einer Umkehrbarkeit der Abfolge, was natürlich bei einer funktionierenden, wohlgeordneten Syntax nicht leicht zu erreichen ist. Innerhalb letzterer herrscht ein festgelegter Ablauf vor, welcher in jeder Sprache zwar verschieden, aber eben deshalb in ihr auch mehr oder minder fix ist. Die Zerstörung der Syntax aber setzt erst die verschiedenen Befreiungsmechanismen ins Werk, die nun im Folgenden beschrieben und besprochen werden.

9 Vgl. u. a. Vogel, Juliane: Anti-Greffologie. Schneiden und Kleben in der Avantgarde. In: Wirth, Uwe (Hg.): Impfen, Pfropfen, Transplantieren. Berlin: Kulturverlag Kadmos 2011, S. 159-172. 
Der Gebrauch des Verbs im Infinitiv macht dieses Verbum seinerseits multifunktional: Es ist vielseitig verwendbar, nicht mehr in Hierarchien oder Bewegungsabläufe eingebunden, alles wird in seiner Ordnung umkehrbar, wird gleichsam in eine grundlegende Simultaneität überführt - auf diesen Begriff werden wir etwas später nochmals zurückkommen. Des Weiteren zielt auch das, was Marinetti als die Abschaffung des Adjektivs bezeichnet, auf eine größere Offenheit des Textes, auf eine Indetermination oder - wenn Sie lieber wollen - auf eine Erhöhung der Unbestimmtheitsstellen im Text im Sinne des Konstanzer Literaturtheoretikers Wolfgang Iser. So ist die Leserschaft gezwungen, diese vielen Stellen selbstständig in einen Sinnzusammenhang - in den je eigenen Sinnzusammenhang - zu bringen. Dass hierbei auch das Adverb nicht übrigbleiben kann, da es ja ebenfalls Modalitäten und Eigenschaften definiert und festlegt, kann nicht überraschen. Marinettis Proklamationen zielen auf eine völlige ,Umkrempelung‘ abendländischer Syntax.

Ein weiterer wichtiger Punkt in seinem futuristischen Zukunftsprogramm ist in dieser Hinsicht zweifellos die Abschaffung der Zeichensetzung. Sie führt gleichsam zur freien Verfügbarkeit der Zeichen auf der Seite, zu einer erhöhten Fähigkeit der Gramma-Textualität, da nun Entwicklungen, Abläufe, Chronologien und Kausalitäten, kurzum: alle logischen Verknüpfungen unterbunden werden, die einzelnen Elemente folgerichtig frei flottieren können. Dass hierbei nun auf die Formelsprache der Mathematik beziehungsweise der Naturwissenschaften zurückgegriffen wird, ist aus dieser Sicht höchstens konsequent, wenn auch nicht in der Radikalität gedacht, in der sich die eigentliche ästhetische Stoßrichtung $\mathrm{zu}$ verwirklichen trachtet. Die Betonung der Analogie auf den verschiedensten Textebenen, wie sie in den weiteren Punkten des Manifests vorgeführt wird, ist eine Fortführung insoweit, als nun die einzelnen Elemente im Grunde ohne ein Tertium comparationis miteinander in direkten Kontakt treten können, sozusagen miteinander kurzgeschlossen werden. Dass es hierbei zu syntaktischen, semantischen und anderen Kurzschlüssen kommen kann, ist aus futuristischer Sicht durchaus erwünscht. Denn jede stringente Logik soll außer Kraft gesetzt werden.

Hieraus ergibt sich die Möglichkeit, alles mit allem zu verbinden, ja Analogien letztlich sogar zum Träger vor allem überraschender, nicht zuletzt auch schockartiger Beziehungen und Effekte zu machen, so dass es im Grunde gar nicht um bloße Analogien geht. Die Beispiele, die Marinetti hierfür angibt, sind übrigens ähnlich schwächlich und zum Teil abgeschmackt wie manche Passsagen seiner sonstigen Texte. Mir scheint es evident zu sein, dass er als Theoretiker ein hellerer Kopf denn als Schriftsteller war! Dass all dies auf eine größtmögliche Umordnung und mehr noch Unordnung zielt, dürfte uns schon von Beginn an klargeworden sein, auch wenn - dies gilt es kritisch einzuwenden - diese Unordnung gerade nicht für die Technikeuphorie des Fliegeramateurs Marinetti gelten 
kann. Denn letztere ist das von diesem Flugbegeisterten auf dem Gebiet von Technik und Technologie ja gerade gefeierte Reich der Ordnung, eine Ebene, auf der sozusagen jedes Schräubchen und Rädchen sitzen muss. Sonst fliegen die tollen Kisten einfach nicht ...

Die Verwendung dieser Technik wird von Marinetti aber gerade in jener Art genauester Ordnung gepredigt, die eben durch ihre Strenge zur größten Unordnung führen kann und in aller Regel auch führt. Durch die militärische, kriegerische Nutzung von Technik wird ein zerstörerisches Element entbunden, dessen destruktive und menschenverachtende Energie der Erste Weltkrieg mit aller wünschenswerten Deutlichkeit und Brutalität unter Beweis stellen sollte.

Von großer Wichtigkeit für unsere Vorlesung ist die Tatsache, dass Marinetti im Technischen Manifest vor allem eine Zerstörung, eine Destruktion des Ich einfordert. Dieses ich, so könnten wir formulieren, war das eigentliche Zentrum, das eigentliche Herzstück der Literatur des 19. Jahrhunderts, das erkaltete Herzblut der Romantik. Kein Zweifel: Das Ich bildete das Zentrum des Impressionismus als Impression im Subjekt, die Potenzierung des Ichs in der Vielfältigkeit und Gleichzeitigkeit der Erfahrungen das Zentrum im Fin de siècle, so dass diese Wendung Marinettis hier auf einen Kernbestand abendländischer Literaturtradition zielt und diese Tradition in der Tat auch zu zerstören sucht. Auch auf dieser Ebene präsentiert Marinetti den eigenen Ansatz mit der Geste einer Befreiung des Menschen von der Verderbnis durch Archive, Bibliotheken und Museen, von der Beeinflussung durch die Psychologie, wobei der italienische Futurist wohl nicht zuletzt auch an die Vertreter des französischen psychologischen Romans gedacht haben mag, die innerhalb des Literaturfeldes Frankreichs eine starke Position einnahmen.

An die Stelle des Ichs muss laut Marinetti nunmehr die Materie treten, die Stofflichkeit, die damit ins Zentrum, in den Mittelpunkt des Kunstwerks rückt. Diese Materie wird der Kunst gleichsam schlagartig und durch Intuition zugänglich, eröffnet sich damit dem Künstler in einer Weise, die von den exakten Naturwissenschaften an Intensität nicht erreicht werden kann. Was Marinetti die lyrische Besessenheit der Materie nennt, wird in der abstrakten Kunst etwa zur unmittelbaren Erfahrbarkeit von Materialien, Stoffen und ihrer Konsistenz: einschließlich aller Federn und Fette eines Joseph Beuys (um einmal einen namhaften Neo-Avantgardisten in dieser Tradition zu nennen). An dieser Stelle wird von Marinetti etwas vorgedacht, was sicherlich die gesamte Kunst und deren Entwicklung betreffen wird, was freilich aber auch die Literatur selbst angeht, deren Bestandteile, Materie und Träger nun ins Zentrum künstlerischer und literarischer Aufmerksamkeit rücken. Insoweit lässt sich Marinettis gewiss noch schemenhaftes Vorhaben durchaus auch als wegweisendes, in die Zukunft gerichtetes futuristisches Programm für die Künste verstehen. 


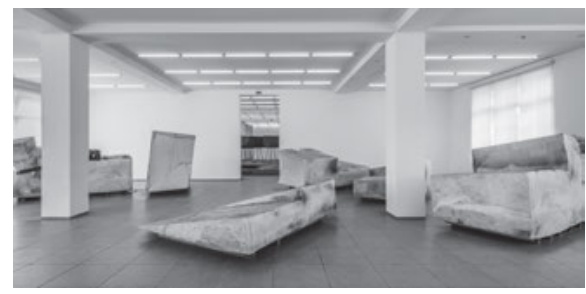

Abb. 32: Joseph Beuys: Unschlitt / Tallow, Installation, 1977.

Bemerkenswert ist freilich, dass Marinetti sein Technisches Manifest wie schon sein Gründungsmanifest allein unterzeichnet und damit nicht kollektiv untermauert hat - im Gegensatz zu vielen anderen seiner manifestartigen Verlautbarungen und auch zu vielen der mehr als zweihundert futuristischen Manifeste, von denen wir heute wissen. Dies mag uns zu der Frage führen, was es denn mit Manifesten insgesamt auf sich hat, eine Frage, der ich in der gebotenen Kürze nachgehen möchte, ist es uns doch (glücklicherweise) nicht möglich, die allein schon für den Futurismus nachweisbaren und eben erwähnten gut zweihundert Manifeste für unsere Vorlesung aufzuarbeiten.

Die historischen Avantgarden waren eine Bewegung der Manifeste, wie Wolfgang Asholt und Walter Fähnders in der Einleitung zu ihrem bereits mehrfach angeführten Band betonten..$^{10}$ Die verschiedenen Ismen der einzelnen avantgardistischen Bewegungen (wie Futurismus, Dadaismus, Surrealismus etc.) konstituieren sich dabei mit Hilfe von Dokumenten, die zu wahren Geburtsurkunden ihrer jeweiligen Bewegungen werden: Manifeste erst bezeugen die ,Geburt' einer neuen Avantgardebewegung! Nicht immer habe es ein großes ästhetisches Werk gebraucht, um eine Bewegung zu kreieren, immer aber ein Manifest, das werbewirksam entworfen sein musste und stets auf unmittelbare Wirkung abzielte.

Im Manifest beweisen die Avantgardistin oder der Avantgardist, in Wirklichkeit eine Vorhut darzustellen - bis hin zur Provokation, zur Geste des Bruchs und öffentlichen Konfrontation. Es gibt die unterschiedlichsten Formen von Manifesten von sogenannten Gründungsmanifesten bis etwa hin zum Anti-Manifest oder auch zu jener Art von Manifest, die wie das Dada-Manifest von 1918 die eigenen Forderungen annulliert. Trotz der Vielgestaltigkeit der Manifeste lässt sich freilich, wie etwa Wolfgang Asholt betonte, in ihnen die Einheit der jeweiligen Avantgarde erkennen, so unterschiedlich auch die Inhalte der je einzelnen Deklamationen und Proklamationen sein mochten. Zumeist spielte die Einlösung der Forderungen des Manifests nicht die entscheidende Rolle, ein durchaus krea-

10 Asholt, Wolfgang / Fähnders, Walter: Einleitung. In (dies., Hg.): Manifeste und Proklamationen der europäischen Avantgarde (1909-1938), S. xv-xxx. 
tives und produktives Problem, das wir im Übrigen schon bei Marinetti hatten durchscheinen sehen: Das Manifest selbst ist bereits ein künstlerischer und revolutionärer Akt an sich und in sich.

Manifeste, so ließe sich mit Wolfgang Asholt und Walter Fähnders behaupten, werden geschrieben, wenn das künstlerische Werk als solches nicht mehr auszureichen scheint. Doch das Manifest selbst ist zugleich bereits Kunstwerk und nicht bloßer Metadiskurs. Die Manifeste des 19. Jahrhunderts, so etwa jene des Symbolismus, hätten zur Kunstautonomie noch beigetragen, indem sie die hermetische Dimension einer bestimmten Kunstauffassung noch betont hätten. Eben dies sei zum Ansatzpunkt für den Bruch der avantgardistischen Manifeste geworden. Denn in der Tat gingen sie einen anderen Weg, der nicht länger jener eines mehr oder minder hermetischen Metadiskurses war.

Ich wäre freilich nicht einverstanden, würde man - wie dies immer wieder geschieht - behaupten, dass man weiter als im Ästhetizismus die Trennung zwischen Kunst und Leben nicht hätte treiben können. Denn dieser Ästhetizismus des Fin de siécle ging durchaus mit der Lebenspraxis eine eigenartig intensive Verbindung ein, denken wir etwa nur an die Figuren des Dandy oder des Bohemien, welche wahrlich zum Inbegriff einer Umsetzung ästhetischer Vorstellungen und Prinzipien in ganz konkretes Leben wurden. Die Manifeste der historischen Avantgarden siedeln sich an der Grenze zwischen Kunstwerk und außerkünstlerischer Wirklichkeit an: Wir könnten sie daher auch als Schwellentexte bezeichnen, wofür auch ihr Grundzug als Mischgattung spricht, also die Verwendung heterogener narrativer und diskursiver Bestandteile, wie wir sie schon im Gründungsmanifest des Futurismus vorfinden konnten. Gerade Marinetti erwies sich als Meister dieser Vermengung unterschiedlichster Textelemente in seinen Manifesten.

Wolfgang Asholt und Walter Fähnders unterscheiden in den von ihnen untersuchten Manifesten zwei unterschiedliche Traditionslinien: Es gebe erstens eine wenig originelle und eher traditionelle Fortführung bestimmter Gepflogenheiten des ,Manifestantismus‘ im Sinne eines Proklamierens und Postulierens, wie wir dies schon gesehen hatten. Die von Marinetti jeweils präsentierten Punkte fallen durch ihre Radikalität oder gar Brutalität auf, nicht aber durch ihren Stil, ihre Sprache oder ihre künstlerische Gestaltung. So bleibe das Verhältnis zwischen Postulat und Form traditionell, eine Tatsache, die sogar für viele DadaManifeste gilt. Auch das erste surrealistische Manifest bewegt sich trotz der Phantasie, der hier auf die Sprünge geholfen werden soll, in recht konventionellen Bahnen.

Gleichwohl komme es gegenüber früheren Beispielen in den avantgardistischen Manifesten insoweit zu einem Bruch, als nun auch narrative Elemente eingebunden würden, die gemäß der beiden Herausgeber dem Genre gänzlich fremd 
seien. Die Konventionen sprengte auch die Gestaltung vieler avantgardistischer Manifeste durch ihr spezifisches Layout, die Verteilung der Wörter auf der Seite oder etwa deren Hervorhebung durch graphische Mittel, so dass man durchaus behaupten dürfe, dass hier eine neue Form literarischer Kommunikation erprobt werden sollte.

Als ein Beispiel nochmals aus dem Bereich des italienischen Futurismus darf ich Ihnen das nicht wenig kriegerische Manifest Sintesi futurista della guerra vom September 1914 einmal vorführen. Ein Manifest, das die kriegerische Ausrichtung und zugleich die avantgardistische Position innerhalb der Kriegsereignisse just jener Gruppe inszenierte, welche auch dieses futuristische Manifest signiert hatte und als eine Art Stoßtruppe aufgefasst werden konnte:

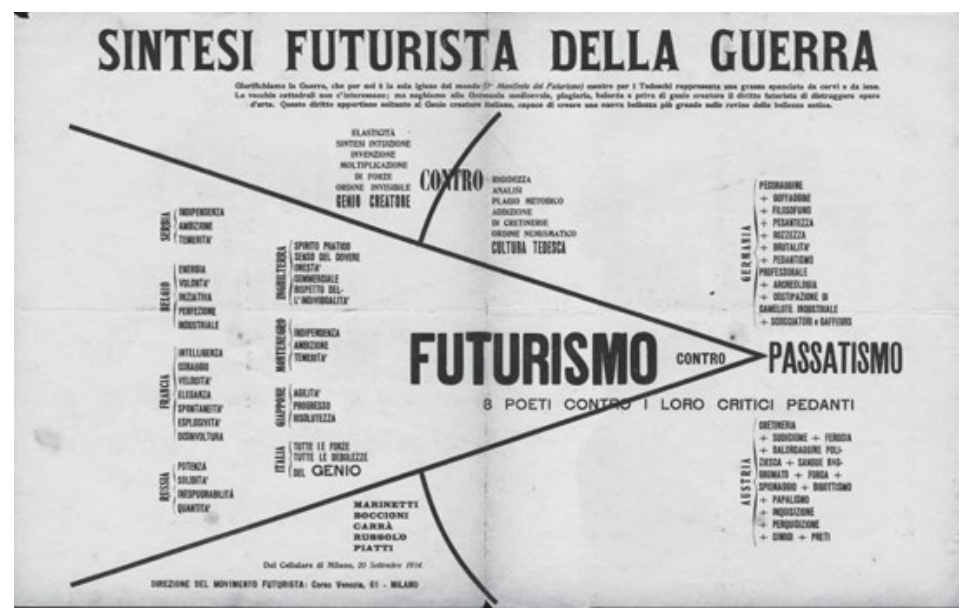

Abb. 33: Filippo Tommaso Marinetti, Umberto Boccioni, Carlo Carrà, Luigi Russolo, Ugo Piatti: Sintesi futurista della guerra, 1914.

Im Kern dieses Manifests, das sich zum Plakatieren bestens eignete, standen die Gegensatzbegriffe „Futurismo“ versus „Passatismo“: Intendiert war mithin eine Wendung gegen alles, was die Vergangenheit repräsentierte. Parallel zu diesem Hauptgegensatz finden sich darunter sogleich „8 Popoli-Poeti“, die gegen ihre „Critici Pedanti“ aufgefahren werden. Auch auf der rein graphischen Ebene stehen die Futuristen in einer vorwärtsstrebenden Avantgarde-Position, welche alles, was die Vergangenheit repräsentiert, gleichsam aus dem Bildausschnitt drängt. Auf der Seite dieses aus dem Bild Gedrängten stehen Deutschland und Österreich mitsamt der sie repräsentierenden herabwürdigenden Attribute. Nein, es war nicht die Zeit für Zwischentöne: Harte Gegensätze mussten aufeinander prallen! 
Dieses Manifest wurde von den militanten Schocktruppen um Marinetti, genauer von Marinetti, Boccioni, Carrà, Russolo und Piatti unterzeichnet, die sich schon durch ihre graphische Anordnung einen besonderen Platz bei der Bekämpfung des Vergangenen und des „Passatismo“ einräumten, all jener Werte also, für die stellvertretend Deutschland und Österreich und die völlig zurückgebliebene Türkei aufgeführt werden. Auf wessen Seite das Kriegspendel ausschlägt, lässt sich unschwer erkennen! So besitzt dieses Manifest auch unverkennbar den Charakter einer politischen Propagandaschrift, die sich in der Nähe zum politischen Agitprop und zur Plakatkunst befindet.

Doch kommen wir nun zur zweiten Traditionslinie: Denn auf der anderen Seite lässt sich eine Art experimentelle Dimension beobachten, insoweit Manifeste der Avantgarde nicht nur auf inhaltlicher, sondern auch formaler Ebene revolutionäre Traditionsbrüche umzusetzen versuchen, so dass man bisweilen von wahren Experimentalmanifesten sprechen könne. Beide Linien des Avantgarde-Manifestantismus beanspruchen für sich Kunstcharakter; und beide versuchen im Übrigen, diesen nicht aus einer Autonomie der Kunst heraus begründet zu sehen. Bisweilen regte sich aber auch ein Unbehagen der Avantgarde an ihren einsträngig und kohärent formulierten Forderungen, die vorgebracht, aber nicht eingelöst wurden: Viele Autorinnen und Autoren waren sich durchaus der Tatsache bewusst, dass sich hieraus ein gewisser Widerspruch ergab, welcher vielen Manifesten zu Grunde liegt.

Gleichwohl bleibt festzuhalten, dass der Manifestantismus während dreier Jahrzehnte nicht nur Italien und Frankreich, sondern ganz Europa und Lateinamerika sowie viele Länder weit darüber hinaus erfasste. ${ }^{11}$ Die Manifeste der unterschiedlichsten Ismen gingen hin und her, wurden zu einer bevorzugten Kommunikationsform im nationalen wie internationalen Literatur- und Kulturbetrieb. Manifeste sind vor allem performative Texte und Performanz ist folglich eine der vorrangigen Bedeutungsebenen eines Manifests. Aus diesem Grunde kommt gerade den Aufführungen und Inszenierungen von Manifesten eine hohe Bedeutung zu.

Dabei zeigt sich zugleich eine auffällige Verschiebung bei der Manifest-Produktion vom einzelnen Individuum zum Kollektiv. Letztlich, so stellten Asholt und Fähnders in ihrer Einführung in die avantgardistische Produktion von Manifesten und Proklamationen fest, sind individuelle Manifeste ebenfalls Ausdruck von Gruppenprozessen oder beheimaten zumindest die Absicht, solche in Gang zu setzen. Insgesamt bleibt festzuhalten, dass es sich bei Manifesten fast immer

11 Vgl. hierzu die Bemerkungen zur Lyrik in Videla de Rivero, Gloria: Direcciones del vanguardismo hispanoamericano. Estudios sobre poesía de vanguardia: 1920-1930. Documentos. Mendoza: Universidad Nacional de Cuyo 2011. 
um Texte als Ausdrucksformen von Bewegungen handelt, für die das Kollektive, die überindividuelle Vereinigung, einen hohen Stellenwert besitzt. Dabei gilt es zu berücksichtigen, dass diese avantgardistischen Kollektive überwiegend männlich waren und man sich ausnahmslos um eine männliche Führungsfigur scharte, welche der Bewegung sozusagen ein Gesicht gab und die Direktiven für die gesamte Gruppe beschloss. Doch auch hier gab es, wie wir noch sehen werden, bemerkenswerte Ausnahmen.

Das Kollektiv ist zugleich die Definitionsinstanz, eine überindividuelle Dimension, die dann vor allem mit dem französischen Surrealismus weiter an Boden und Bedeutung unter der leitenden Hand André Bretons gewinnen wird. Er verstand es auf meisterhafte Weise, die Spannungen zwischen herausragendem Individuum und künstlerisch-literarischer Gruppe immer wieder zu lösen und zugleich fruchtbar zu machen. Die kollektiven Unterschriftenlisten sind von enormer Bedeutung nicht zuletzt auch für die jeweilige Situation im literarischen und künstlerischen Feld, dessen aktuelle Problematik gleichsam nominell, auf Ebene von Namen, gespiegelt wird. Auf derartige Fragen der Gruppenbildung werde ich zu einem späteren Zeitpunkt erneut zurückkommen, kommt hier doch das Umschlagen des Latenten ins Manifeste überdeutlich zum Ausdruck.

Lassen Sie mich als letztes futuristisches Manifest einmal keines aus der Feder Marinettis vorführen, sondern eines aus der Hand eines berühmten futuristischen Malers. Dieser bemühte sich nicht nur um die Frage der Simultaneität die im Grunde für jede Art von Malerei eine grundlegende Fragestellung ist -, sondern versuchte darüber hinaus, andere Formen sinnlicher Wahrnehmung in seine Malerei, aber auch in seine Manifestationskunst einzubauen. Uns bleibt keine Zeit, das relativ umfangreiche Manifest vom 11. August 1913 in all seinen Details zu untersuchen; ein Manifest, das übrigens in streng marinettistischer Tradition die einzelnen Punkte hübsch ordentlich aufeinanderfolgen ließ und alles brav durchnummerierte. Aufschlussreich ist vor allem der Schlussabschnitt dieses Dokuments eines der Mitbegründer des Futurismus, den ich Ihnen nicht vorenthalten möchte:

Wir futuristischen Maler erklären, dass die Töne, Geräusche und Gerüche im Ausdruck der Linien, der Volumen und der Farben Gestalt annehmen, genauso wie Linien, Volumen und Farben in der Architektur eines Musikwerkes Gestalt annehmen. Unsere Bilder werden also auch die bildnerischen Äquivalente der Töne, Geräusche und Gerüche des Theaters, der Music-Hall, des Kinos, des Bordells, der Bahnhöfe, der Häfen, der Garagen, der Kliniken, der Fabriken usw. zum Ausdruck bringen. ${ }^{12}$

12 Carrà, Carlo Dalmazzo: Die Malerei der Töne, Geräusche und Gerüche. In: Asholt, Wolfgang / Fähnders, Walter (Hg.): Manifeste und Proklamationen, S. 58. 


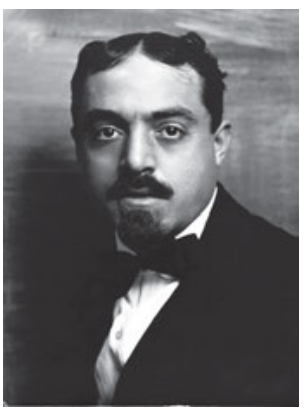

Abb. 34: Carlo Carrà (Quargnento, Provinz Alessandria, 1881 Mailand, 1966).

Was in dieser Schlusspassage des Manifests von Carlo Dalmazzo Carrà auffällt, ist zum einen dessen explizit kollektiver Charakter, selbst wenn es nur von einem einzigen Maler unterzeichnet wurde. Doch dieser wusste sich an diesem Punkte durchaus eins mit einer Vielzahl italienischer Malerkollegen, die unter dem Banner des Futurismus längst in die nationalen und internationalen Diskussionen eingegriffen hatten und oftmals auch bei den futuristischen „serate“ teilnahmen. Diese netten Abende konnten nicht selten in wilde Happenings mit wüsten Beschimpfungen des Publikums - das aber auch einmal mitgebrachtes Obst und Gemüse in Mailand auf die anwesenden Künstler warf - ausarten. Gerade diese häufig in Gruppen auftretende Schar, die von Marinetti gut gesteuert und organisiert an die Öffentlichkeit trat, dürfte für die weitreichende Wirkung der futuristischen Kunstauffassung wesentlich mitverantwortlich gewesen sein. Die Futuristen schufen beim Publikum und in der Öffentlichkeit eine nicht selten brodelnde Atmosphäre, die nichts mehr mit einem introspektiven Kunsterleben $\mathrm{zu}$ tun haben wollte.

Auf inhaltlicher Ebene ist von großer Bedeutung, dass sich der italienische Maler Carrà hier explizit auf das Zusammenwirken der unterschiedlichsten Künste, Sinneswahrnehmungen und Lebensbereiche bezieht. Diese werden gleichsam in ein Gesamtkunstwerk überführt und gebündelt, in welchem der Synästhesie eine entscheidende Relevanz zukommt. Die inter- und transmediale Verbindung der Töne, Geräusche und Gerüche spricht unmittelbar die musikalische und allgemeiner noch die akustische Sinneswahrnehmung doppelt an, verbindet sie mit dem Olfaktischen und natürlich auch mit der Malerei selbst, der Domäne dieses futuristischen Künstlers, und ihren ausdrucksstarken Farben.

Der Literatur kommt in diesem Sinnenspektakel eine sicherlich transmedial verbindende, keineswegs aber mehr zentrierende Stellung im Konzert der Künste $\mathrm{zu}$. Von hoher Bedeutung sind die phonotextuellen Elemente, die gerade auch bei der Beziehung zur Lautkunst und Lautpoesie eine bis heute überraschende Funktion ausüben. Denn Literatur als Klang ist in unserer Gegenwart leider in den 
Hintergrund ihrer Betrachtung gerückt: Sie ist durch die akustische Ausblendung über das stille Lesen ${ }^{13}$ und die ,Entklanglichung' von Literatur nahezu unbewusst geworden. Es ist aber zweifellos ein großes Verdienst der Futuristen im Besonderen und der Avantgarden im Allgemeinen, diesen phonotextuellen Aspekt wieder in den Vordergrund gerückt oder zumindest wieder bewusst gemacht zu haben. Die Literatur also klingt! Gerade die Lyrik ist - schon von ihrer etymologischen Wortgeschichte her - eine klingende, schwingende, vibrierende Kunstpraxis und nicht nur die Verteilung von Buchstaben in mehr oder minder geordneter Weise auf einer weißen Seite. Dies ist ein Aspekt von Literatur und Lyrik, der übrigens durch die historischen Avantgarden ganz wesentlich wieder befördert und ins allgemeine Bewusstsein gehoben wurde.

Was haben wir uns unter einer solchen Art von Klangkunst vorzustellen? Da mir keine historischen Aufnahmen der italienischen Futuristen zur Verfügung stehen, möchte ich an dieser Stelle auf einen großen deutschen Vertreter der historischen Avantgarden zurückgreifen, auf Kurt Schwitters. Einige wenige biographische Daten zu ihm mögen uns an dieser Stelle unserer Vorlesung freilich genügen!

Der deutsche Schriftsteller und Bildende Künstler Kurt Schwitters wurde in Hannover am 20. Juni 1887 geboren und starb im englischen Kendal am 8. Januar 1948. Man darf in diesem Tausendsassa durchaus einen der einflussreichsten Künstler des 20. Jahrhunderts erkennen. Nach dem Abitur studierte Schwitters Kunst, später auch Architektur in Hannover, Berlin und an der Dresdener Kunstakademie. Nach seiner Heirat im Jahr 1915 wurde er 1917 kurzzeitig zum Militärdienst eingezogen, aber aufgrund seines labilen Gesundheitszustands und epileptischer Anfälle bald schon wieder freigestellt. Er lebte während der zwanziger und dreißiger Jahre abwechselnd in Hannover und Norwegen, wohin er 1937 endgültig emigrierte, nachdem er in Deutschland als „entartet“ verfemter Künstler angesehen wurde. Schwitters interessierte sich für die zeitgenössischen Entwicklungen in Kunst und Literatur, beschäftigte sich etwa mit Kubismus oder später Dadaismus, trat aber selbst niemals der dadaistischen Bewegung bei. 1940 floh er vor dem deutschen Einmarsch nach England, wo er bis zu seinem Tode lebte.

13 Zur Geschichte des stillen Lesens vgl. Ette, Ottmar: LiebeLesen. Berlin - Boston: De Gruyter 2020, wo in verschiedenen Kapiteln die Geschichte und Geschichten der Leserevolutionen besprochen werden, vgl. insbes. S. 93-103. 


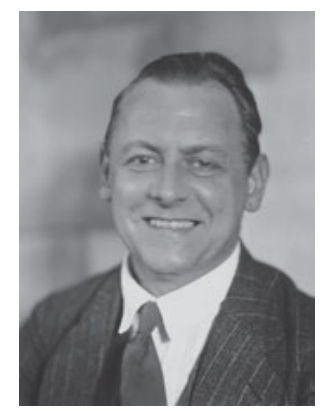

Abb. 35: Kurt Schwitters (Hannover, 1887 - Kendal, Cumbria, England, 1948).

Ohne jemals in eine feste Gruppe integriert gewesen zu sein, war Kurt Schwitters zweifellos einer der vielseitigsten Künstler der historischen Avantgarden, der vor allem durch Collagen in allen Bereichen der Künste, von Literatur und Theater über Musik und Bildende Kunst bis hin zu Design und Werbung, experimentierte. In Abgrenzung zu anderen Richtungen der Moderne prägte er für sein künstlerisches Vorgehen das Kennwort „Merz“, ein ,zufälliges‘ Schnipsel aus „Commerzbank“, das er 1918 als Vereinigung von Kunst- und Nichtkunst als „Gesamtweltbild“ definierte und als sein Erkennungszeichen nutzte. Er blieb in seinen Aktivitäten vielen Dadaisten verbunden, ging zugleich aber sehr eigene Wege.

Im Gegensatz zu ihnen verstand er sein eigenes Tun durchaus als Kunst; Schwitters Bemühungen galten zeit seines Lebens der Schaffung eines großen Gesamtkunstwerks. Im Zeichen von „Merz“ operierte er namentlich mit literarischen Collagen, Unsinnpoesie, Zahlen- und Lautgedichten, surreal grotesken Dialogen und arbeitete immer wieder experimentell mit unterschiedlichsten typographischen Gestaltungen, die ihm einen festen Platz in der Kunstwelt seiner Zeit sicherten. Er experimentierte mit verschiedensten „objets trouvés“ und fügte die unterschiedlichsten alogischen Objektstrukturen literatur- und bildtechnisch zusammen. Von 1923 bis 1932 gab Schwitters die Zeitschrift $M E R Z$ unregelmäßig heraus, an der Hans Arp, Theo von Doesburg und andere Avantgardisten mitarbeiteten und in der erstmals 1932 Schwitters Ursonate erschien, ein Beispiel absoluter Dichtung als Einheit von Klang, Text und Bild, ganz jenen Prämissen folgend, wie sie Carlo D. Carrà in seinem futuristischen Manifest formuliert hatte. Berühmt machte ihn das Anti-Liebesgedicht An Anna Blume von 1919, das er auf Litfaßsäulen plakatierte.

Ich möchte Ihnen am Beispiel zweier Selbstaufsprachen von Kurt Schwitters einmal vorführen, wie Gedichte im Mund ihres Autors und in unseren Ohren klingen. Dabei handelt es sich zunächst um das erwähnte, wohl bekannteste Gedicht Schwitters', Anna Blume, das gleichsam eine Liebeserklärung an die Sprache und an das Spiel mit ihr darstellt. In einer zweiten Aufsprache wird 


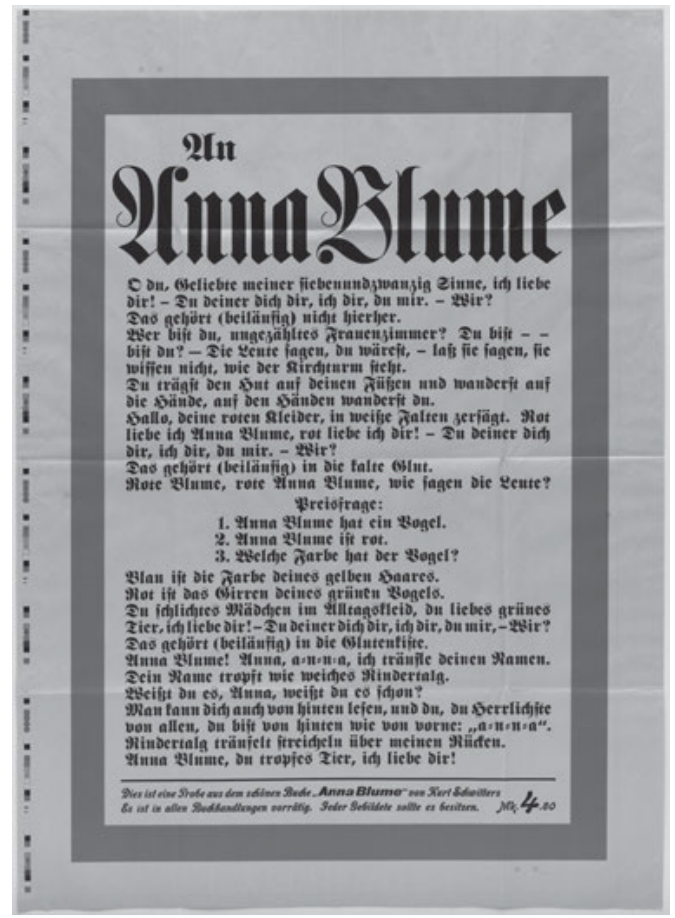

Abb. 36: An Anna Blume, Plakat, um 1920.

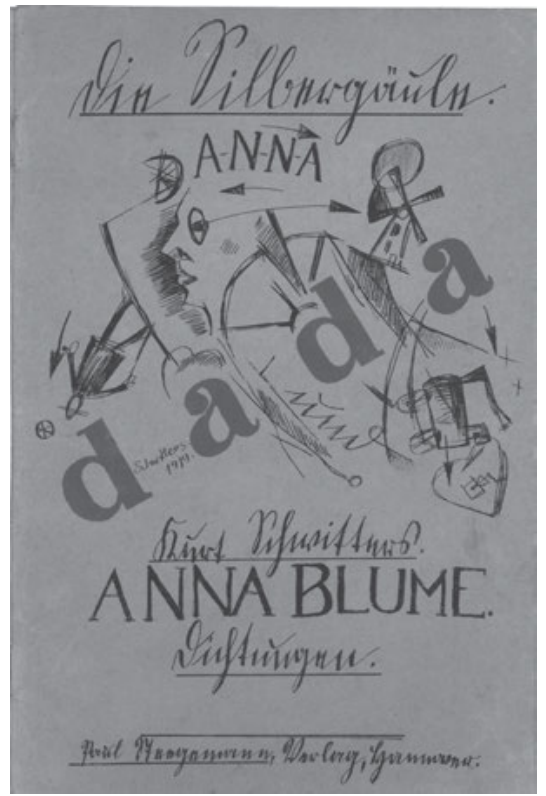

Abb. 37: Cover der Erstausgabe von Kurts Schwitters' Anna Blume. Dichtungen, 1919. 
der Übergang zum ,reinen` Lautgedicht ohrenfällig, zumal die hier eingespielte, angespielte Kunst nicht mehr vor allem die Literatur (in Form des Gedichts), sondern die Musik ist, wodurch die dionysische Kraft im Sinne Nietzsches direkt im Lautgedicht in relativ strenger, geschlossener Form, jener der Sonate, verwendet und adaptiert wird. Ich wünsche Ihnen viel Spaß bei diesem Hörvergnügen, das ich Ihnen zunächst einmal ohne jede schrifttextliche Unterstützung gönnen will! Die Aufnahme entstand im Mai 1932, zu einem Zeitpunkt also, der es Schwitters vor der Machtergreifung der Nationalsozialistischen deutschen Arbeiterpartei noch letztmalig ermöglichte, in Deutschland künstlerisch tätig zu sein. ${ }^{14}$ Danach wurde von den Nationalsozialisten die gesamte künstlerische Avantgarde entweder ausgeschaltet, gleichgeschaltet oder - wie Schwitters - ins Exil getrieben.

Doch wenden wir uns nun auch dem Text zu:

An Anna Blume

Oh Du, Geliebte meiner 27 Sinne, ich liebe Dir!

Du, Deiner, Dich Dir, ich Dir, Du mir --- wir?

Das gehört beiläufig nicht hierher!

Wer bist Du, ungezähltes Frauenzimmer, Du bist, bist Du?

Die Leute sagen, Du wärest.

Laß sie sagen, sie wissen nicht, wie der Kirchturm steht.

Du trägst den Hut auf Deinen Füßen und wanderst auf die Hände,

Auf den Händen wanderst Du.

Halloh, Deine roten Kleider, in weiße Falten zersägt,

Rot liebe ich Anna Blume, rot liebe ich Dir.

Du, Deiner, Dich Dir, ich Dir, Du mir, --- wir?

Das gehört beiläufig in die kalte Glut!

Anna Blume, rote Anna Blume, wie sagen die Leute?

Preisfrage:

1. Anna Blume hat ein Vogel,

2. Anna Blume ist rot.

3. Welche Farbe hat der Vogel?

Blau ist die Farbe Deines gelben Haares,

Rot ist die Farbe Deines grünen Vogels.

Du schlichtes Mädchen im Alltagskleid,

Du liebes grünes Tier, ich liebe Dir!

Du Deiner Dich Dir, ich Dir, Du mir, --- wir!

Das gehört beiläufig in die --- Glutenkiste.

Anna Blume, Anna, A---N---N---A!

14 Tonaufnahme online noch abrufbar unter https://www.youtube.com/watch?v=U2TIVTHzFT0 (letzter Zugriff 07.06.2020). 
Ich träufle Deinen Namen.

Dein Name tropft wie weiches Rindertalg.

Weißt Du es Anna, weißt Du es schon,

Man kann Dich auch von hinten lesen.

Und Du, Du Herrlichste von allen,

Du bist von hinten, wie von vorne:

A-----N-----N-----A.

Rindertalg träufelt STREICHELN über meinen Rücken.

Anna Blume,

Du tropfes Tier,

Ich-----liebe------Dir! ${ }^{15}$

Was für ein wunderbares Liebesgedicht! Es versammelt viele der Diskurselemente der Liebe, die Roland Barthes in seinen Fragments d'un discours amoureux versammelte und welche den Liebesdiskurs im Abendland wesentlich strukturieren. ${ }^{16}$ Und zugleich führt es diesen Liebesdiskurs an seine absurden Grenzen, macht sein Umkippen in eine Welt des Absurden deutlich - um gerade daraus wieder sein Potenzial als Liebeserklärung, als Vorbringen der Formel des „Ich liebe Dich“ verballhornt zu beziehen. Denn das Formelhafte, das laut Roland Barthes Erzwungene der Liebesformel des „Je t’aime“ wird geschickt umgangen, indem sich ein scheinbar umgangssprachlicher Fehler in den Liebesschwur eingeschlichen hat und ihn gerade dadurch individualisiert. So ist die Liebe auch und gerade im „Anti-Liebesgedicht“ tierisch überwältigend!

Auch Schwitters Sonate mit Urlauten aus dem Jahr 1932, die ich Ihnen hier in einer Aufnahme von Mai 1932 präsentieren darf, ${ }^{17}$ steht für die ganze sinnliche Laut- und Klangkunst der Avantgarden beispielhaft ein. Nach diesem Ohren- und Augenschmaus fällt es leichter zu begreifen, in welch vielfältiger medialer und intermedialer Weise die historischen Avantgarden - und die italienischen Futuristen als erste unter ihnen - versuchten, die Grenzen zwischen den einzelnen künstlerischen Disziplinen aufzulösen und das Gefängnis der Worte der Literatur aufzubrechen. Und dies erfolgte gleichzeitig in die unterschiedlichsten Richtungen. Genau auf diesem weiten Feld künstlerischer Erneuerung - und gerade nicht im politischen Bereich der Affinität zum Faschismus - ist die eigentlich emanzipatorisch relevante Kraft der italienischen Futuristen und dieser frühen avant-

15 Schwitters, Kurt: Anna Blume (1919). In: Boer, Klaus (Hg.): Kurt Schwitters: Anna Blume. Texte zu Anna Blume und Merz. Grafrath: Boer 2019, S. $11 \mathrm{f}$.

16 Vgl. Barthes, Roland: Fragments d'un discours amoureux. Paris: Seuil 1977; vgl. hierzu auch Ette, Ottmar: LiebeLesen, S. 60-92.

17 Tonaufnahme online abrufbar unter https://www.youtube.com/watch?v=6X7E2i0KMqM (letzter Zugriff 07.06.2020) 
gardistischen Bewegung zu sehen. Ich kann Ihnen natürlich an dieser Stelle in einem Buch die Beziehung zur Sinneswahrnehmung des Geruchs nicht bieten, es wäre denn, sie nähmen jetzt den Band ganz dicht an ihre Nase und atmeten in der Falz noch die Spuren des Klebstoffes ein, der beim Binden des Buches verwendet wurde. Es handelt sich aber zweifellos um ein Sensorium, das nicht zuletzt auch im Theater oder bei den öffentlichen „serenate“ aufgerufen und performativ in Szene gesetzt werden kann. Derartige gemeinschaftliche Happenings waren ja eine der bevorzugten künstlerischen Ausdrucksweisen der Futuristen.

Damit aber möchte ich zur Betrachtung einer weiteren Gattung übergehen, die für die italienischen Futuristen von großer Bedeutung war, gerade weil sie sich hier ebenfalls nicht an die tradierten engen Grenzen hielten. Denn in sehr grundlegender Weise wird bei ihnen das Theater zum Gesamtkunstwerk, zu einem intermedialen Ereignis und mehr noch Spektakel. Wie gesagt, ein solches waren insbesondere die futuristischen Abende, die ,serenate futuriste“, die häufig von der Polizei geschlichtet und beendet werden mussten, da sie allzu häufig in wilden Handgemengen und Schlägereien endeten. Marinetti mietete dazu meist die Bühnen von Theatern großer Städte an, wo dann die unterschiedlichsten Künste und Künstler zu Wort kamen und wo insbesondere der Rezitationskunst - vergessen wir nicht: Filippo Tommaso Marinetti war Rezitationskünstler, lange bevor er den Futurismus begründete! - eine besondere Rolle zukam.

In einem solchen Rahmen wurde nicht nur das Gründungsmanifest des Futurismus rezitiert, sondern auch eine Vielzahl anderer experimenteller Texte ausprobiert. Die Futuristen wurden zunehmend zu theatralischen PerformanceKünstlern und erfüllten damit jene Dimensionen künstlerischen Ausdrucks, wie wir sie etwa in der postmodernen Performance-Kunst und nicht zuletzt auch in kulturellen Praktiken außereuropäischer Kulturen finden können. Ziel war bei den ,serenate“ nicht zuletzt die Aktivierung der Zuhörerschaft, des Publikums und zwar nicht in einem vorwiegend rezeptiven Sinne, sondern als aktivem Publikum mit all seinen Sinnen und in gewisser Weise bar jeder abendländischen Vernunft.

Diese Zuhörerschaft reagierte bisweilen auf die gezielten Publikumsbeschimpfungen so gehaltvoll, dass der soeben zitierte futuristische Maler Carlo D. Carrà einmal angesichts der ihm entgegenfliegenden Auswahl preiswerter Gemüsesorten seinem Publikum entgegenschrie, man möge sie doch nicht mit Tomaten, sondern mit Ideen bewerfen, „ihr Idioten“! Das Theatralische ist folglich eine Praxis, die im Umfeld des Futurismus und der Avantgarden weit über das im herkömmlichen Sinne verstandene Theater oder gar das „Theater als moralische Anstalt“ hinausging. Es zielt vor allem auf die Überwindung der vierten Wand, also der Rampe zwischen Bühne und Publikum oder - wie wir nun gewitz- 
ter sagen können - zwischen Kunst und Leben. Die Aktivierung des Publikums war ein zentraler Programmpunkt des Futurismus wie auch der historischen Avantgarden insgesamt insoweit, als hier die Grenze zwischen Kunst und Lebenspraxis vielleicht nicht immer überwunden, aber doch begehbar und subvertierbar gemacht werden konnte. Im Theater, in den „serenate“, war die Kunst im Leben handgreiflich zu spüren.

Aber das Theater war bereits zu einem früheren Zeitpunkt Bestandteil futuristischer Praxis. Marinetti selbst hatte 1905 ein Stück in französischer Sprache mit dem Titel Roi Bombance geschaffen, ein Theaterstück, in dem die Anleihen an Alfred Jarrys Ubu Roi überdeutlich sind. Dieser Rückgriff auf Jarry zeigt sich im avantgardistischen futuristischen Theater Marinettis auch später noch; doch wird schon an dieser Stelle einer der Urväter des futuristischen Theaters sichtbar, eben der Begründer der Pataphysik Alfred Jarry. Nicht umsonst kommen seine Theatergebilde aus der Schule der Marionetten; und nicht umsonst werden die futuristischen Bühnendichter, die ihrerseits Manifeste verfassten, in der Entwicklung ihrer Theaterkunst zunehmend Elemente der Automatisierung und des Maschinellen wie Seriellen miteinbauen. Die künstlerischen Umsetzungsformen waren dabei sehr unterschiedlich: So wählte man etwa die ästhetische Form sich marionettenhaft bewegender Schauspieler, den Einsatz von Marionetten, aber auch von Spielpuppen und Automaten oder die hochtechnisierten Formen eines Theaters, das die futuristische Ausrichtung an der Maschine eindrucksvoll in Szene setzte.

Viel wäre an dieser Stelle dazu zu sagen, doch können wir uns hier einiges sparen, da wir im unmittelbaren Anschluss diesen Vorläufer der historischen Avantgarden namens Alfred Jarry mitsamt seiner Theaterkonzeption kurz besprechen werden. Für den Augenblick mag es genügen, dass wir uns vom futuristischen Theater an dieser Stelle einen Gesamteindruck verschaffen, zumindest was die schrifttextliche Seite angeht. Die Macht der Performance, die Gewalt der Inszenierung müssen Sie sich unmittelbar vorstellen!

Entscheidend ist, dass das futuristische Theater - und allen voran Marinetti - eine neue Theaterform entwickelte, die durch ihre besondere Kürze hervorsticht: die futuristische „sintesi“. Unter diesen kurzen Theatersynthesen, von denen Marinetti selbst wohl die einflussreichsten verfasste, dürfen wir verdichtete Theaterformen verstehen. Diese entwickeln und präsentieren die Themen der Simultaneität, der Gleichzeitigkeit des eigentlich Ungleichzeitigen, der „compenetrazione“, also des wechselseitigen Ineinanderwirkens und SichDurchdringens in bestimmten verdichteten Augenblicken, des „dinamismo“, also einer erhöhten Dynamik und Geschwindigkeit, aber auch des Gemütszustandes in ganz futuristischer Tradition (wenn dieser Ausdruck erlaubt ist). Spannend sind diese „Theatersynthesen“ nicht zuletzt auch deshalb, weil sie 
uns eine nanophilologische Praxis ${ }^{18}$ vor Augen führen, das Verfassen von Kurzund Kürzesttexten, die in sich als Fraktale, als miniaturisierte Modelle, von ungeheurer künstlerischer Durchschlagskraft sind. Zugleich weisen sie auch auf künstlerische Praktiken der „Erfindung“ neuer ästhetisch-literarischer Kurz- und Kürzestformen ${ }^{19}$ im Verlauf der sich anschließenden hundert Jahre voraus.

In diesen „Theatersynthesen“ oder Kürzeststücken geht es vorzüglich um die schnelle Bewegung, die Geste, das Evozieren alogischer Zustände, mithin um Entwicklungen und Bewegungen, die nicht durch eine Ursache-Wirkung-Konnexion miteinander verbunden werden. Man könnte bisweilen auch von fast traumartigen Sequenzen sprechen, die gleichsam unser Imaginäres direkt ,anzapfen“ oder doch zumindest ansprechen. Hierbei kommt den literarischen Unbestimmtheitsstellen eine hohe Bedeutung $\mathrm{zu}$, lassen sie doch der Interpretation durch bestimmte futuristische Schauspielgruppen, die Italien seit etwa 1914 unsicher machen, und vor allem auch dem Publikum fast alle Wege einer Deutung offen. In ihrer Kürze sind sie radikal polysem.

Diese Form des Theaters verlangte bisweilen der Maschinerie des Bühnenbetriebes vieles ab, was kleinere Theatergruppen kaum bieten konnten, zielte gar auf eine grundlegende Mechanisierung der Welt der Schauspieler, so dass letztere gänzlich überflüssig gemacht werden konnten und durch Automaten ersetzt wurden. Doch gerade auch das Ineinanderwirken verschiedener Künste war entscheidend: Die Bühnenbildner, Beleuchter, Maskenbildner und viele mehr hatten bei den Futuristen eine wirklich hohe Kunst zu entwickeln, um die Synästhesien und das Ineinanderwirken von Literatur, gesprochenem Wort, Farbe, Plastizität, Körperlichkeit und dynamischer Bewegung, aber auch von Geruch und Geräusch voranzutreiben. Neu war die Idee des Gesamtkunstwerks keineswegs, finden wir sie doch in voller Blüte bei einem Richard Wagner. Aber die Radikalität futuristischer Synästhesien beeindruckt doch bis heute!

Ich möchte Ihnen an dieser Stelle einmal ein derartiges Stück - und zwar aus der Feder von Filippo Tommaso Marinetti - vorführen, ein Stück, das programmatisch den Titel Simultaneità trägt. Auch hierbei kommen wieder Akzente wie schon im vorhergehenden Manifest zum Tragen, die der hohen Kultur der Literatur eine Massenkultur und Alltagskultur an die Seite stellen, welche von

18 Vgl. zu Theorie und Praxis der Nanophilologie Ette, Ottmar: Nanophilologie. Literarische Kurzund Kürzestformen in der Romania. Tübingen: Max Niemeyer Verlag 2008.

19 Vgl. Ette, Ottmar / Sánchez, Yvette (Hg.): Vivir lo breve. Nanofilología y microformatos en las letras y culturas hispánicas contemporáneas. Madrid - Frankfurt am Main: Iberoamericana 2020. 
den automatisierten Gesten des Vaudevilles bis hin zur Bordellkultur oder Industriekultur des beginnenden 20. Jahrhunderts reicht. Gleichzeitig bekommt dieses Stück natürlich auch eine zusätzliche ästhetische Dimension als Lesedrama, das mit der Lyrik den Vorteil des raschen Einschlagens, der unmittelbaren Wirkung durch die Einheit der Wahrnehmung teilt:

Salon. - Die rechte Wand besteht aus einem großen Bücherregal. - Zur Linken ein großer Tisch. - an der linken Wand einfache Möbel und eine Tür. - An der hinteren Wand ein Fenster, durch das man es draußen schneien sieht, und eine andere Tür, die sich zur Treppe hin öffnet.

Um den Tisch herum, unter einer Lampe mit Lampenschirm in dämmrigem, leicht grünlichem Licht, sitzt die Familie des Angestellten: DIE MUTTER näht, DER VATER liest Zeitung, DER SECHZEHNJÄHRIGE SOHN macht Schularbeiten, DER SOHN VON 10 JAHREN macht auch Schularbeiten, DIE FÜNFZEHNJÄHRIGE TOCHTER näht.

Ganz nah an der Bibliothek ein prächtiger Schminktisch, hell erleuchtet mit Spiegeln und Kandelabern, voller Fläschchen und Tiegel und sämtlicher Utensilien, die eine äußerst feine Dame braucht. Ein besonders intensiver Scheinwerfer leuchtet den Schminktisch aus; an ihm sitzt eine junge KOKOTTE, sehr schön, blond, in einem luxuriösen Morgenmantel. Sie hat aufgehört, sich zu kämmen und beginnt, Gesicht, Armen und Händen die letzten Tupfer zu geben, aufmerksam assistiert von einer strengen KAMMERZOFE in aufrechter Haltung.

Die Familie sieht diese Szene nicht.

DIE MUTTER zum VATER: Willst du die Rechnungen überprüfen?

DER VATER: Das schau ich mir später an. Er liest weiter.

Stille. - Alle gehen mit Selbstverständlichkeit ihren Beschäftigungen nach. DIE KOKOTTE macht sich weiter zurecht, unsichtbar für die Familie.

DIE KAMMERZOFE geht zur hinteren Tür, als hätte sie es klingeln gehört, und öffnet einem BOTEN, der der KOKOTTE einen Blumenstrauß und einen Brief überreicht. DIE KOKOTTE riecht an den Blumen und liest den Brief. - DER BOTE geht respektvoll grüßend ab.

DER SECHZEHNJÄHRIGE unterbricht seine Arbeit und schaut zum Fenster hinaus: Es schneit immer noch ... Was für eine Stille!

DER VATER: Dieses Haus liegt wirklich sehr einsam. Wir ziehen nächstes Jahr um ...

DIE KAMMERZOFE geht wieder zur hinteren Tür, als hätte es nochmals geklingelt, und läßt eine junge MODISTIN herein, die auf sie zugeht und so tut, als trüge sie in ihrem leeren Karton einen wunderschönen Hut. DIE KOKOTTE tut so, als probiere sie den Hut vor dem Spiegel auf, wird ärgerlich, weil er ihr nicht gefällt, und legt ihn beiseite. Dann gibt sie dem Mädchen Trinkgeld und fordert es mit einem Wink auf, zu gehen. Das Mädchen geht grüßend ab.

DIE MUTTER scheint etwas auf dem Tisch zu suchen, steht plötzlich auf und geht zur linken Tür hinaus, als wollte sie etwas holen.

DER VATER erhebt sich und geht zum Fenster. Er bleibt vor dem Fenster stehen und schaut hinaus.

Nach und nach schlafen die drei Kinder am Tisch ein.

DIE KOKOTTE verläßt ihren Schminktisch und nähert sich langsam, mit vorsichtigen Schritten, dem Tisch. Sie nimmt die Rechnungen, die Hefte und Handarbeiten und wirft alles achtlos unter den Tisch. 
DIE KOKOTTE: SCHLAFT!

Sie geht langsam zum Schminktisch zurück und lackiert sich weiter ihre Fingernägel. ${ }^{20}$

Nach Lektüre dieses gesamten Theaterstückes reibt man sich in einem ersten Augenblick verwundert die Augen. Denn Marinettis „Synthese“ ist zunächst einmal kaum an Banalität zu übertreffen. Wir haben es mit in der Tat geradezu klischeehaften Bildern zu tun: eine gutbürgerliche Familie, im trauten Heim versammelt, vielleicht auch ein wenig kleinbürgerlich, selbst wenn die Existenz eines Dienstmädchens oder das Bücherregal dagegen spricht, kontrastiert mit einer schönen und herausgeputzten jungen Frau, die von Beginn an als Kokotte bezeichnet wird und für käufliche Liebe steht. Die Familie ist rund um ihren Patriarchen bei ihren verschiedenen Verrichtungen geradezu rollengemäß versammelt in einem Haus wohl am Rande einer Stadt. Der Ehemann liest Zeitung, die Kinder machen ihre Schulaufgaben, beziehungsweise das Mädchen ihre Nadelaufgaben, während die Mutter sich um alles kümmert. Gutbürgerlicher und zugleich klischeehafter geht es nicht!

Doch in dieses Bild hineinprojiziert sehen wir als Zuschauer dieser Guckkastenbühne ein anderes Geschehen, welches die einzelnen Figuren aber selbst nicht sehen können. Der Titel Simultaneität erläutert gleichsam didaktisch dieses Geschehen, das man nun auf die unterschiedlichsten Arten - und genau hierin liegt der Traditionsbruch) mit der erstgenannten Szenerie in Beziehung setzen kann. Denn die nicht weniger klischeehafte Vertreterin eines horizontalen Gewerbes der Luxusklasse macht sich fertig für ihre Aufgaben: sie bereitet sich also vor auf die nächtlichen Unternehmungen ihres anti-bürgerlichen Lebens, welches freilich in der Antibürgerlichkeit eben dieses bürgerliche Leben wiederum bedient und stützt. Die Kokotte richtet sich hübsch her für die Männer, die sich offenkundig bei ihr melden.

Die Intention dieser „Theatersynthese“ ist ganz offensichtlich. Denn natürlich muss man sich fragen, in welchem Zusammenhang beide Szenen miteinander stehen. Dass sie nicht völlig voneinander getrennt sind, sondern sich in irgend einer Weise aufeinander beziehen, wird schon daran deutlich, dass sie in der Einheit von Raum, Zeit und Handlung re-präsentiert werden. Gingen wir aber von zwei verschiedenen, sich im Grunde nicht überlagernden Diegesen aus, die nur ineinandergeblendet wären, dann wird diese Trennung am Ende durch die Aufforderung der Kokotte über die Grenze hinweg zerbrochen. Das Publikum mag rätseln, ob der Ehemann und die Kokotte miteinander zu tun haben, eine

20 Marinetti, Filippo Tommaso: Simultaneità. In Schnapp, Jeffrey T. (Hg.): Teatro. Bd. 2. Mailand: Mondadori 2004, S. 543. 
in der Zeit selbstverständlich naheliegende heterosexuelle Beziehung, wäre für die Zeitgenossen doch eine Relation zwischen Kokotte und Ehefrau eher nicht vorgesehen. Freilich könnten wir aus heutiger Sicht eben diese Beziehung bei einer Neuinterpretation dieses Theaterstückes beispielsweise in den Vordergrund rücken. Dies stünde uns frei! Sie sehen, man könnte mit den von diesem verdichteten Stück dargebotenen Konstellationen wunderbar spielen, um jeweils verschiedenartige Beziehungsmuster hervortreten zu lassen.

Zugleich sollten wir uns gerade bei Marinetti vor Augen halten: Selbst das hochgradig Konventionelle ist letztlich aus einer Sicht freier Bezüglichkeit ein Bruch! Denn gezeigt wird genau dies: Dass eigentlich vom Sujet her keine teure Theateraufführung erforderlich ist. Dieses miniaturisierte Theaterstück bildet in seinem Verhältnis zu den damaligen Konventionen des bürgerlichen Theaters der Zeit einen Bruch, der jenen Bruch vorwegnimmt, welchen das spätere Theater des Absurden mit dem Rückgriff auf ähnlich banale Themen - wie etwa jenes des endlosen Wartens in Samuel Becketts Waiting for Godot oder eines enger werdenden Raumes in Eugène Ionescos Les Chaises vorwegnimmt. Die gespenstische Atmosphäre auf der Theaterbühne ergibt sich auch und vor allem aus der Banalität des Dargestellten, das ,eigentlich` doch nicht die Hauptsache sein dürfte, hier aber nun in der Tat in den Mittelpunkt des Theaterstückes gestellt wird. Für die Zeitgenossen war es durchaus eine Provokation - oder hatte zumindest das Zeug dazu.

Marinetti sagte über sein Stück, er habe hier „die gleichzeitige Durchdringung des Lebens einer bürgerlichen Familie mit einer Kokotten inszeniert“, wobei die Kokotte hier kein „Symbol, sondern eine Synthese (sei) aus Gefühlen von Luxus, von Unordnung, von Abenteuer und Verschwendung, die als quälende Sehnsucht oder Schmerz in den Nerven aller Personen, die um den Tisch herum sitzen, existieren“. ${ }^{21}$ Damit zielte er gleichsam auf ein Essentielles im Menschen, das gegen die Einordnung in eine bürgerliche Gesellschaft und die Unterordnung unter hierarchische Strukturen rebelliert. Es handle sich laut Marinetti um eine „absolut autonome theatralische Synthese“, welche „weder dem bürgerlichen Leben noch der Halbwelt ähnlich“ sei. ${ }^{22}$ Dabei meint diese Art ästhetischer Synthese keineswegs eine Form gesellschaftlicher Symbiose: Beide Welten sind noch immer strikt voneinander getrennt. Es wird lediglich eine simultane Ko-Präsenz erzeugt.

Eine solche Synthese wolle nur sich selber ähnlich sein. Damit berührte Marinetti den Aspekt einer Selbstähnlichkeit, der für jegliches fraktale Modell von entscheidender konzeptioneller Bedeutung ist. Die Fraktalität seiner Theatersyn-

21 Ebda., S. 545.

22 Ebda. 
these kann daher im Sinne eines miniaturisierten Modells verstanden werden, das keineswegs nur eine spezifische Familie oder eine bestimmte gesellschaftliche Klasse in einem konkreten Land repräsentiert, sondern insgesamt die Gesellschaft und vielleicht mehr noch die gesamte Menschheit bedeutet. Denn ein fraktales Modell zielt gerade auf Grund seiner Miniaturisierung stets auf Totalität, einen Makrokosmos an Elementen ab.

Zugleich handle es sich um eine absolut dynamische theatralische Synthese, so Marinetti weiter, der stets seine Stücke als Ausfluss seiner Theorien beschreibt und letztlich damit auf diese theoretische Dimension reduziert. Auch an dieser Stelle bestätigt sich, dass der italienische Futurist wohl ein besserer, weitsichtigerer Theoretiker als ein durch seine literarischen Ausdrucksmittel faszinierender Schriftsteller war. Im Sinne des ersteren wird die absolute Bewegung von Raum und Zeit erzielt, eine simultane Durchdringung von zwei Räumen und verschiedenen Zeiten, wie wir sie in diesem Kürzeststück gespiegelt sehen. Denken wir dabei immer wieder auch an das im Eingangsbereich unserer Vorlesung mehrfach genannte Bild von Spiegeln, die sich in ihren Bewegungen wechselseitig spiegeln ...

Wir können in Simultaneität also die ganzen zentralen Kategorien erkennen, die Marinetti für seine Theaterkunst futuristischer Synthesen reklamiert. Die vielleicht wichtigste von allen ist die Behauptung, eben nicht mehr von der außersprachlichen Wirklichkeit abzukupfern, sondern gleichsam die Mimesis selbst absolut zu setzen, ähnele eine Synthese doch nur der Synthese selbst oder der von Synthesen erzeugten und hervorgebrachten Kunstwelt. Verdichtung, so scheint mir, ist hier im Verbund mit Miniaturisierung ein weiteres Gebot dieser Art nanophilologischen Schreibens. Marinetti war mit seinen Kürzesttexten auf dem Gebiet des Theaters zweifellos ein Pionier, auch wenn wir gleich noch sehen werden, dass er für seine Vorstellungen und Ideen sehr wohl Modelle besaß, die von großer Wirkkraft und von starkem Einfluss auf die nachfolgenden Avantgarden waren.

Wir können uns anhand dieser theatralischen Synthese sehr gut vorstellen, dass die einzelnen Schauspieler auch durch Sprechpuppen oder Marionetten zu ersetzen waren. Denn den Theaterfiguren haftet im Grunde keine psychologische Tiefe an, sie sind nicht wirklich individuiert; vielmehr entsprechen sie einer Verdichtung von Attributen, die bestimmten Repräsentanten und Repräsentantinnen der europäischen Gesellschaften vorbehalten waren. Dass sich hier wiederum Beziehungen gerade auch zum symbolistischen Theater herstellen lassen, steht außer Frage!

Auf das symbolistische Theater bezog sich in kritischer Distanzierung aber auch ein anderer Künstler, der im Frankreich der Jahrhundertwende einen entscheidenden Schritt vorwärts gegangen war und den wir in unserer Vorlesung 
über das Fin de siécle zwar ungern, aber aus didaktischen Gründen wohlüberlegt nicht berücksichtigt hatten: Alfred Jarry. Ich glaube nicht, dass man auf diese große Figur der Literatur und Bühne verzichten kann, will man die ästhetische Entwicklung im Verlauf des 20. Jahrhunderts auch in ihren Tiefenströmungen begreifen. Gehen wir daher kurz einen zeitlichen Schritt zurück, um einen ästhetischen Schritt vorwärts zu tun!

Bevor wir uns in der gebotenen Kürze mit diesem mir sehr ans Herz gewachsenen französischen Schriftsteller und seinem Werk beschäftigen, in welchem man eine Vorläuferschaft für die historischen Avantgarden sowie das Theater des Absurden, für das Theater der Grausamkeit und selbst noch für Oulipo, das Ouvroir de littérature Potentielle, erkennen kann, möchte ich Ihnen zunächst einige biographische Daten zum Werk des verehrten Begründers der Pataphysik mit an die Hand geben.

Alfred Henri Jarry wurde am 8. September 1873 als Sohn eines wohlhabenden Kaufmanns in Laval (Frankreich) geboren. Während seiner Schulzeit komponierte der junge Jarry bereits seine später berühmten Comédies en vers et en prose, die erhalten blieben. Am Lycée de Rennes arbeitete damals ein Physiklehrer namens Hébert, den man als „Père Heb“ oder „Père Ebé“ titulierte, der für die Schüler zum Inbegriff des Grotesken und Gegenstand erster Szenen und Theaterstücke wurde. Eine Fassung eines solchen Stücks seines Schulfreundes Charles Morin wurde von Alfred Jarry umgearbeitet und als Marionettenstück am Jahreswechsel 1888/89 auf dem Speicher der Familie zur Aufführung gebracht. 1890 erfolgte eine Inszenierung mit den Marionetten des Théatre des Phynances in der Privatwohnung Jarrys: Es handelt sich um die erste Fassung des späteren Stückes Ubu Roi. Weitere Kompositionen und Stücke entstehen: Es sind Handschriften, die später etwa unter den Surrealisten zirkulieren sollten. Immer wieder werden diese „Urszenen' aufgeführt und von Jarry akribisch verändert. Die beruflichen Träume des jungen Mannes verwirklichen sich währenddessen nicht: Mehrfach scheitert er an der Aufnahme in die prestigeträchtige Ecole Normale Supérieure. Und auch ein Philologiestudium an der Sorbonne wird er niemals abschließen.

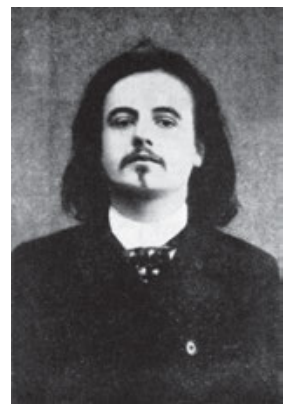

Abb. 38: Alfred Jarry (Laval, Département Mayenne, 1873 - Paris, 1907). 
Doch die Pariser Literaturszene wird erstmals auf Jarry aufmerksam und literarische Freundschaften bilden sich. 1894 erscheint Jarrys erstes Buch. Dann markiert das Jahr 1896 eine für Alfred Jarrys Werk wichtige Epoche: die Zusammenarbeit mit Lugné-Poe, dem Direktor des Thêâtre de l'EEuvre. Eine erste schriftliche Fassung von Ubu Roi erscheint in einer literarischen Monatsschrift und wenig später im Mercure de France. Am 10. Dezember wird Ubu Roi dann im Théâtre de l'Euvre mit der Musik von Claude Terrasse uraufgeführt. Die Folge ist einer der berühmtesten Theaterskandale der französischen Literatur: An die berühmte Auftaktszene mit dem Ausruf „Merdre“, verballhornt für das französische „Scheiße“, schließen sich tumultartige Szenen und ein längeres Handgemenge an: Die bürgerliche Kritik reagiert pikiert, Jarry muss sich erklären. Doch fortan ist er weit über die Theaterzirkel hinaus ein bekannter Autor. Jarry beginnt mit der Ausarbeitung verschiedener $U b u$-Zyklen, die freilich bis auf seine Schulzeit zurückgehen. Mit seinen Heldentaten und Ansichten des Doktor Faustroll gelingt ihm der Gründungstext dessen, was Jarry als die „Pataphysik“ bezeichnet, jener urkomischen Wissenschaft von den erfundenen, imaginären Lösungen. Die Tumulte bei der Uraufführung und die Konzeption eines „Collège de Pataphysique“ lassen Jarry für die Nachwelt wie einen Avantgardisten avant la lettre erscheinen.

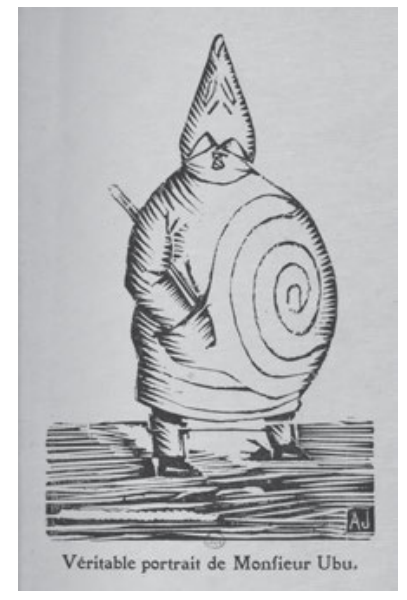

Abb. 39: Alfred Jarry: Porträt „König Ubu“.

Zum Markenzeichen Jarrys wurde sein berühmtes Fahrrad, ${ }^{23}$ mit dem er überall unerwartet auftauchte: Ungezählte Photographien zeigen ihn auf diesem Gefährt, das zugleich ein Zeichen unbedingter Modernität war. In sein Leben flossen

23 Vgl. hierzu Siepe, Hans T.: Metropolis, Cyclopolis und Tour de France. Kleine Bemerkungen zum Motiv von Stadt und Fahrrad in der Avantgarde. In: Klein, Wolfgang / Fähnders, Walter / 


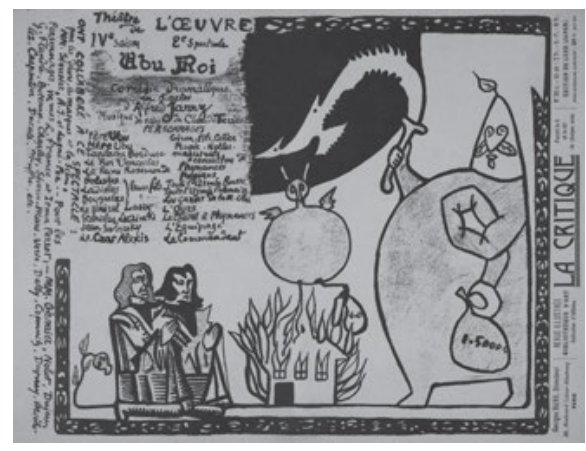

Abb. 40: Plakat der Uraufführung von Ubu Roi am Théâtre de l'GEuvre, 10. Dezember 1896.

zunehmend sprachliche Ticks und Redensarten seiner literarischen Hauptfiguren ein: Schon für ihn war die gesamte künstlerische Tätigkeit darauf abgestellt, Kunst und Leben miteinander zu verschmelzen und das eigene Leben in ein Kunstwerk $\mathrm{zu}$ verwandeln.

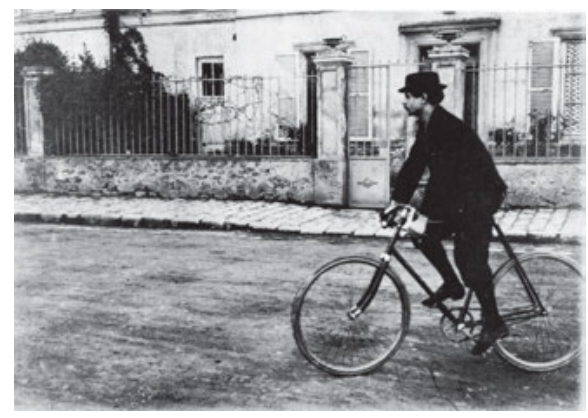

Abb. 41: Alfred Jarry auf seinem Fahrrad.

Dieses Kunstwerk hatte nur ein kurzes Leben: Alfred Jarry erkrankte schwer, machte aber weiter seine Scherze ebenso über die Krankheit wie über die „merdecins“, die ihn behandelten. Doch sein Humor konnte ihn nicht mehr retten: An Tuberkulose erkrankt, starb Jarry im Alter von vierunddreißig Jahren am 1. November 1907 im Hôpital de la Charité zu Paris. Zeit seines Lebens war er ein literarischer Außenseiter gewesen; doch seine Wirkung und sein Nachruhm machten ihn zu einer einflussreichen Kunstfigur, die bis heute mit dem berühmten Fahrrad als Bürgerschreck durch die Straßen von Paris radelt und für den lustvoll-radikalen Aufbruch in Richtung Avantgarde steht.

Grewe, Andrea (Hg.): Dazwischen. Reisen - Metropolen - Avantgarden. Festschrift für Wolfgang Asholt. Bielefeld: Aisthesis Verlag 2009, S. 443-451. 
Ohne auf Jarry und seine Theaterästhetik allzu detailliert eingehen zu können und ohne seinen bereits erwähnten Roman Le supermâle zu berücksichtigen, der insbesondere auf Marinettis ersten Roman Mafarka le futuriste großen Einfluss ausübte, möchte ich Ihnen doch eine Reihe von Facetten seines Werks vorstellen. Diese sind für die Fragestellung unserer Vorlesung, also die Traditionslinie zu den historischen Avantgarden und zunächst zum italienischen Futurismus, von Relevanz. Von zentraler Bedeutung in Jarrys gesamtem Schaffen ist sein erwähntes Drama in fünf Akten, Ubu Roi, das in Paris - wie bereits erwähnt - am 10. Dezember 1896 im Théâtre de l'EEuvre von Lugné-Poe uraufgeführt wurde.

Aurélien-Marie Lugné-Poe dürfen wir uns als die vielleicht schillerndste Figur der Pariser Theaterwelt vorstellen, die gleichsam das Off-Theater der damaligen Zeit in der französischen Hauptstadt entscheidend prägte und immer wieder neue Stücke auch nicht-französischer Autoren - allen voran etwa Ibsen - präsentierte. Ubu Roi ist es durchaus noch anzumerken, dass das Stück ursprünglich auf einen Schülerstreich zurückging, der freilich weitreichende theaterästhetische Folgen hatte und das Leben von Jarry entscheidend prägen sollte. Ubu alias Hébert war zu Beginn nicht viel mehr als eine Ulk-Figur; doch diese sollte monumentale Züge gewinnen, die ausgehend vom ursprünglichen Stück Les Polonais transhistorische Ausmaße annahmen. Denn König Ubu geriet immer mehr zu einem Diktator, Populisten und Feigling, der seine Macht unbeschränkt zur Anwendung bringt, solange er keinen empfindlichen Widerstand spürt. Er ist eine Figur, welche die Zeiten und Kulturen durchläuft und für das bevorstehende 20. Jahrhundert, aber auch für unsere Zeit geradezu prophetischen Charakter besitzt. Denn auch jetzt mangelt es an Populisten nicht!

In seiner grotesken Komödie, die ursprünglich für Marionetten ohne jede psychologische Tiefe ausgelegt war, verwandelte sich König Ubu mit Hilfe unendlich vieler Eingriffe und Veränderungen langsam in eine geradezu mythische Gestalt, die zugleich etwas Absurdes und Archetypisches besitzt. Der ruhmreiche Begründer der Pataphysik, die Jarry gerade auch in sein Leben zu implementieren nicht müde wurde, schuf ein Theaterstück, das zwar seiner Entstehungsgeschichte nach noch immer in Polen verortet war, wo es auch heute nicht an Rechtspopulisten mangelt, doch betonte sein Schöpfer mit Recht, dass dieses Stück im Grunde überall und nirgends spiele.

Lassen wir daher zunächst einmal den Autor des Stückes zu Wort kommen! Denn schon am 10. Dezember 1896 hatte Alfred Jarry unmittelbar, bevor sich der Vorhang im Théâtre de l'Euvre hob, klar gemacht, dass die Handlung eben in Polen spiele, das heiße also nirgendwo! In einer weiteren Präsentation heißt es dann: 


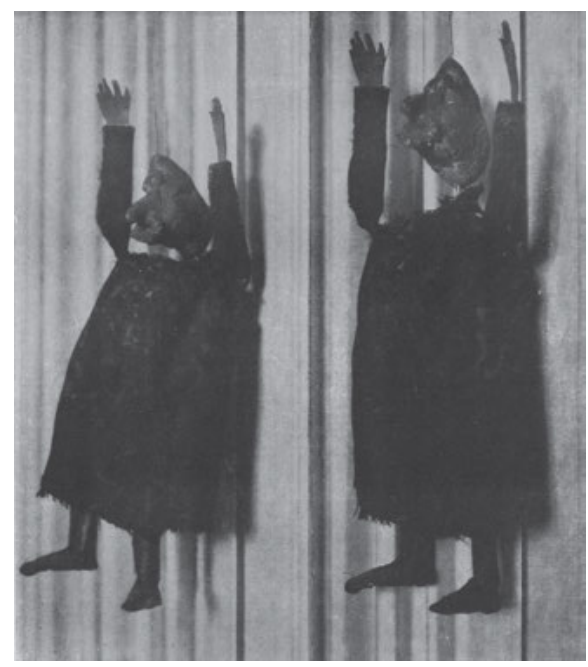

Abb. 42: Marionetten der Uraufführung von Ubu Roi.

Nach dem Vorspiel einer Musik mit allzu viel Blech, um weniger zu sein als eine Fanfare, einer Musik, die just das darstellt, was die Deutschen eine „Militärkapelle“ nennen, enthüllt der Vorhang ein Bühnenbild, das gerne ein Nirgendwo repräsentierte, mit Bäumen zu Füßen der Betten, mit weißem Schnee unter einem schönen blauen Himmel, wobei die Handlung also in Polen vonstatten geht, einem ausreichend legendären und auseinander genommenen Land, um dieses Nirgendwo gut darstellen zu können, zumindest nach einer wahrscheinlichen franko-griechischen Etymologie, weit entfernt, ein fragliches Irgendwo. [...] Nirgendwo ist überall, und ganz besonders das Land, in dem man sich befindet. Aus eben diesem Grunde spricht Ubu Französisch. Aber seine unterschiedlichen Fehler sind keine französischen Laster bloß, denen Kapitän Bordure zuneigt, der Englisch spricht, oder die Königin Rosamunde, die in einem Dialekt aus dem Cantal kauderwelscht, oder die Masse der Polen, die alle kopfnäseln und grau in grau gekleidet sind. Sieht man darin unterschiedliche Satiren, dann macht der Ort der Bühne daraus die verantwortungslosen Interpreten.

Herr Ubu ist ein abstoßendes Wesen, darin ähnelt er uns allen (von unten). ${ }^{24}$

Wir ersehen aus dieser Passage bereits zum einen, dass Ubu Roi überall angesiedelt sein könnte und tatsächlich auch ubiquitär ist. Eine Beschränkung und Reduktion auf bestimmte nationale Grenzen gibt es nicht. Zum anderen ist $\mathrm{Ubu}$ Roi aber auch jedermann, wir alle, jeder einzelne, jede einzelne von uns. Ubu ist abscheulich, und eben darum uns so ähnlich, wie Jarry es formuliert. Im Grunde ist in diesen Wendungen schon ein Element vorhanden, das in der Internationalität der historischen Avantgarden und insbesondere im Manifestantismus der Züri-

24 Jarry, Alfred: Ubu Roi. Notes et dossier de Laurent Tiesset. Paris: Editions Chemins de tr@ verse 2011, S. 10: Autre présentation d'Ubu Roi. 
cher Dada-Bewegung voll zur Geltung kommen wird: Ubu kennt keine Grenzen; gerade das Ubiquitäre von Ubu ist abscheulich und damit grenzenlos!

Drittens wird aber auch deutlich, dass dem Bühnenbild und der Musik eine wichtige Rolle zukommen: Sie sind keineswegs bloße ,Beilagen'. In der Tat ist Ubu Roi trotz aller Wichtigkeit des Sprechens und der Jarry'schen Wortkreativität ein Theaterstück, das nicht auf ein reines Worttheater reduzierbar ist, sondern alle möglichen Sinneswahrnehmungen und Ausdrucksformen mit einschließt. Ubu Roi ist ein Schritt weg vom spezifisch literarischen Theater und hin zu dem, was man später und bis in unsere Zeit als das „totale Theater“ bezeichnet hat, das mit allen Sinnen und Wahrnehmungsformen des Menschen spielt. Die Banalität und Absurdität der Handlung ist dabei ein Element, das in der Folge - beispielsweise von Marinetti und den Futuristen - sehr stark wiederaufgenommen und modifiziert werden wird, wobei die Satire gegen das Bürgertum, gegen Anpassung und Konvention, gegen Normalität und Moral, die durchgängige Angriffslinie bilden wird. König Ubu ist von seinem ersten Bild, von seinem ersten Wort an ein heftiger Angriff auf alles Überkommene.

$\mathrm{Zu}$ Beginn des Stücks ist Ubu, der Ex-König von Aragon, noch nicht König von Polen, sondern ein hochdekorierter Vertrauensoffizier des polnischen Königs. Bei seinem ersten Erscheinen auf der Bühne löste er jenen bereits erwähnten Theaterskandal aus, und zwar ebenso durch sein äußeres Erscheinungsbild wie durch die berühmten zwei Silben, die als erstes über seine Lippen kommen: „Merdre“ oder auf Deutsch vielleicht „Schreiße“. Alfred Jarry stellt in wenigen Worten klar, wie sich die Geschichte ausgehend von der Eingangsszene, von der ersten Szene des Theaterstückes, entwickeln wird. Denn König Ubu wird sich durch die Welt massakrieren, rücksichtslos, bestialisch und zugleich feige, an bürgerlichen Normen und der Normalität immer orientiert: stets auf seinen Wanst und dessen Wohlbefinden wie auch auf die Befriedigung der unteren Teile des Körpers, wie es Jarry formulierte, aufmerksam achtend. Doch sehen wir uns zunächst einmal die erste Szene des Fünfakters an:

VATER UBU: Schreiße.

MUTTER UBU.: Oh! Das ist ja was Hübsches, Vater Ubu, Ihr seid ein reichlich großer Schlingel.

VATER UBU: Dass ich Euch nicht erschlag', Mutter Ubu.

MUTTER UBU: Nicht ich bin es, Vater Ubu, ein andrer ist's, den Ihr ermorden müsstet. VATER UBU: Bei meiner grünen Kerze, ich verstehe nicht.

MUTTER UBU: Wie denn, Vater Ubu, seid Ihr's zufrieden mit Eurem Schicksal?

VATER UBU: Bei meiner grünen Kerze, Schreiße, Madame, gewiss doch, ich bin zufrieden. Man wär's mit weniger: Kapitän der Dragoner, Vertrauensoffizier des Königs Venceslav, ausgezeichnet mit dem Orden des Roten Adlers von Polen und ehemaliger König von Aragon, was wollt Ihr mehr? [...] 
MUTTER UBU: Was hindert Dich daran, die gesamte Familie zu massakrieren und Dich an ihre Stelle zu setzen? [...]

VATER UBU: Ja, wahrlich! Und hernach was? Habe ich nicht einen Arsch wie alle anderen? MUTTER UBU: An Deiner Stelle würde ich diesen Arsch auf einem Thron installieren. Du könntest Deine Reichtümer unendlich vergrößern, sehr häufig Bratwürste essen und durch die Straßen auf Karossen fahren. [...]

VATER UBU: Ah! Ich gebe der Versuchung nach. Du Schreißluder, Du Luderschreiße, treff' ich ihn jemals am Rande eines Waldes, dann wird er ein übles Viertelstündchen erleben. ${ }^{25}$

Auch wenn König Ubu oder Vater Ubu noch mehrfach Angst (seine berühmte „trouille“) bekommt und später auch Mutter Ubu für alles verantwortlich zu machen sucht, bedarf es doch nur einiger weniger Worte und Verlockungen seiner Frau, um den Scheißkerl Vater Ubu vom Königstreuen zum Königsmörder mutieren zu lassen. Vater Ubu ist unendlich feige, aber unendlich ist auch seine Grausamkeit, solange er nicht selbst bedroht ist und seine sadistische Mordlust ungestraft an anderen vollziehen und ausleben kann. Denn dank dieser Habsucht und Grausamkeit kann er trotz seiner Feigheit über mehr Geld und über größere Würste verfügen, seine Instinkte und Gelüste befriedigen und die ganze Menschheit um sich herum dem Schicksal, der Katastrophe und dem Untergang ausliefern. All dies ficht ihn nicht an! Ist dies nicht eine wunderbare Vorwegnahme all der monströsen Diktatorenfiguren, die das 20. Jahrhundert hervorgebracht hat; und dies nur der Herrschsucht und des Größenwahns wegen? Alfred Jarry hat mit den Mitteln seiner provozierenden Kunst den Tyrannen ein Denkmal gesetzt.

Denn die Mordgelüste eines Bürgers in der Rolle des Diktators sind schnell geweckt: Der dünne Firniss der Zivilisation über den Urinstinkten der Spezies ist rasch ab und die moralische Dimension des Menschen erscheint als Draperie und Dekor. Menschen erscheinen überhaupt als bloße Marionetten bestimmter Instinkte, die ihr gesamtes Handeln regeln: Schnell scharen sie sich hinter einem beliebigen Diktator und begehen ungesühnte Verbrechen. Sie sind letztlich Maschinen, die keine größere psychologische Tiefe kennen, sondern nur die abgrundtief absurde Präsenz ihrer Gelüste und Triebe leben. Hinter der Komik und der unstillbaren Lust auf Schalk und Verulkung lauert in Alfred Jarrys Theaterfigur etwas zutiefst Dunkles, Abgründiges, für die gesamte Menschheit Fatales, das sich auf diesem Planeten überall ereignen kann und de facto überall ereignet hat. Vor König Ubu ist niemand sicher, er ist ganz einfach ubiquitär!

Nun, die Handlung im Theaterstück schreitet erwartungsgemäß voran: Nichts kann Ubu aufhalten. Nachdem Monsieur Ubu die polnische Königsfamilie massakriert und fast vollständig ausgerottet hat, bricht eine kurzfristige Epoche der

25 Jarry, Alfred: Ubu Roi, Acte premier, Scène première, S. 18 ff. 
Glückseligkeit mit dem polnischen Volke an, das jedoch schon bald aus denselben Beweggründen zunehmend brutaler angefasst und rücksichtslos massakriert wird. Ubus Günstling Bordure überredet den russischen Zaren zum Krieg gegen den aragonesischen Usurpator: Ubu muss gegen seinen Willen in den Krieg ziehen. Der Thronaspirant Bougrelas stürzt als einziger lebendig gebliebener Angehöriger der polnischen Königsfamilie die Ubus vom Thron. Doch die Feigheit rettet Ubu im Krieg das Leben: Zusammen mit seiner Frau gelingt ihm die Flucht in sein Heimatland Frankreich. Auch schon in Ubu Roi spielt der Krieg die entscheidende Rolle als Vernichtungsmaschinerie; doch ein Lob des Krieges - wie später bei den italienischen Futuristen - kann man bei Alfred Jarry nicht erkennen. Er lässt keinen Zweifel daran, dass die Kriegsmaschine von den größten Feiglingen betätigt wird, die an ihrer Stelle andere zur Aufopferung in den Krieg schicken.

Schon früh verstanden die Zeitgenossen, wie sehr sich Alfred Jarrys Ubu Roi gegen die Normen und Konventionen der im Grunde brutalen bürgerlichen Gesellschaft richtete, wie sehr hier die Selbstzufriedenheit, Geilheit und Gewissenlosigkeit einer nur scheinbar stabilen Gesellschaft gegeißelt wurde, der nur etwas am eigenen Wohlbefinden lag, gleichsam am Fressen möglichst vieler Würste. Psychische wie physische Vorgänge werden direkt auf die Bühne übertragen, freilich neben den psychologischen Aspekten vor allem auch die schiere Körperlichkeit der Figuren Jarrys. Es ist der groteske Körper, der immer wieder im Rampenlicht steht. Nicht umsonst beginnt alles mit einer Fäkalszene. Und hatte König Ubu letztlich nicht einen Arsch wie jeder andere?

Diese groteske, letztlich aber alltägliche Körperlichkeit spielt von der ersten Szene, von der Exkrementierung des Inneren an, eine ganz entscheidende Rolle. Es gab Aufführungen, in denen dem ersten Wort „merdre“ mehrere Minuten des Schweigens folgten, in denen die einzelnen Figuren in ihrem Dekor und durch ihre schiere Körperlichkeit wirkten. Dabei ist das Stück, ganz in der Tradition des symbolistischen Theaters, durchsetzt von intertextuellen Zitaten und Anspielungen auf andere Theaterstücke und Autoren, wobei hier Shakespeare und Rabelais zweifellos eine besonders wichtige Rolle zukommt. Dies ist keineswegs zufällig, spielt doch nicht nur bei Rabelais' Pantagruel, sondern auch bei William Shakespeare die Körperlichkeit der Theaterfiguren eine bedeutungsvolle und nie gänzlich ausleuchtbare Rolle.

So hat man etwa in der Forschungsliteratur zu Ubu Roi mehrfach festgestellt, dass König Ubu eine Travestie von König Lear, Mutter Ubu von Lady Macbeth ist oder doch so aufgefasst werden kann. Das große Stück Jarrys, an dem der Künstler seit seiner Schulzeit immer wieder arbeitete und das er in verschiedenen Zyklen ebenso lustvoll wie aufopferungsvoll erweiterte, ist ein mit allen Wassern der Literatur gewaschenes und zugleich gewitztes Stück Theatergeschichte, das wie in einer Synthese, einem miniaturisierten Modell, nicht zuletzt auch europäische 
Theater- und Literaturgeschichte bietet. Insofern bietet es nanophilologische Bezüge, die sehr wohl auf die Theatersynthesen Marinettis vorausweisen.

Stellen Sie sich die erste Szene des ersten Aktes von Ubu Roi einmal plastisch vor in einem wirklichen Theaterablauf, vielleicht sogar in einer Inszenierung, die Sie selbst in die Theaterwirklichkeit umsetzen müssten! Bedenken Sie dann, welch geringer Wert schon in dieser Eingangsszene, die doch die Exposition darstellt und daher das Theaterpublikum über die vergangenen Ereignisse und die aktuellen Vorgänge aufklären muss, noch irgendwelchen Erläuterungen oder didaktischen Hinführungen zukommt! An dieser Stelle bleibt nur der Hinweis darauf, dass sich mehrere intratextuelle „continuations“ bei Jarry selbst anschließen, der seinem Stoff etwa schon in Ubu enchaîné oder Ubu in Ketten 1899 - die Uraufführung erfolgte im Jahre 1900 - wiederum neue Perspektiven gab. Rund um die literarisch hochverdichtete Figur Ubu, in welcher doch noch immer der Schülerstreich lebendig blieb, ließ sich in der Tat eine ganze literarische Welt, ja die Totalität von Welt entfalten.

Wie sehr auch immer die Rollen Ubus wechseln, stets ist die Brutalität, Grausamkeit und Absurdität dieser grotesken Figur das leitmotivisch wiederkehrende zentrale Element eines faszinierenden Theaterspektakels. In $\mathrm{Ubu}$ cocu, also $\mathrm{Ubu}$ als Hahnrei, tritt die Titelfigur als Doktor der Pataphysik auf, der sein Gewissen stets in einem Koffer mit sich schleppt. Was für ein wunderbares Bild, das vorausweist auf die gewissenlosen Mörder im 20. (und 21.) Jahrhundert, die ihr Gewissen an der Uniform-Garderobe abgegeben hatten und frei davon handeln und töten. In diesem Stück wird das „Enthirnungslied“ gesungen, das bereits vorausweist auf die Ästhetik eines Marinetti, eine Metaphorik der Zermantschung und grausamen Zerstörung also, die wir nicht nur bei den Futuristen, sondern später auch bei Boris Vian wiederfinden werden. Alfred Jarrys Ideen begeisterten gerade nach den Grausamkeiten zweier Weltkriege eine europäische Leserschaft, die versuchte, die Spuren des Kommenden in den Literaturen der Jahrhundertwende aufzufinden, die also den Versuch unternahm, die vergangene Zukunft für sich zu entdecken. Und da stand Alfred Jarry mit seiner grotesken Figur an allen Wegkreuzungen.

Jarrys Theaterkonzeption zielte auf eine unmittelbare Konfrontation mit dem zeitgenössischen Publikum, lebte von ihm und dessen Reaktionen auf die schockierenden und provozierenden Elemente der Stücke. Es ist ein Publikum, das zugleich in seinen Erwartungen ent-täuscht und angeklagt wird, hält man ihm doch einen Zerrspiegel vors Gesicht, der durchaus mit dem Verfahren eines anderen Autors des Fin de siècle in Verbindung zu bringen wäre: Ramón del ValleInclán und seiner Ästhetik der „esperpentos“, der verzerrenden und absurden Spiegelungen. Mir scheint, dass gerade diese Beziehung zu einem wegweisenden spanischen Autor viel von der Problematik aufzeigt, welche die Kontinuitätslinien von Fin de siècle und Avantgarde hervortreten lassen und die Ästhetik des 
Bruches im Sinne von Peter Bürger nicht zuletzt als eine bewusste, vorsätzliche Inszenierung erscheinen lassen.

Alfred Jarrys Theaterstück, das mit den vielsagenden Worten Vater Ubus „Gäbe es kein Polen, dann gäbe es auch keine Polen“ und damit einer kalauernden „vérité de Lapalisse“ endet, wurde vom Autor in einigen Szenen auch zeichnerisch begleitet und gestaltet. Zeichnungen, die zeigen, wie genau sich Jarry eine groteske Gestalt vorstellte, die höchste Anforderungen an Bühnenbildner und Dekorateure, vor allem aber auch an Maskenbildner stellte. Es handelt sich kaum noch um Menschen: Père Ubu erscheint vielmehr als ein „fantoche“, eine gespensterhafte Puppe, die mehr von ihren Instinkten gehandelt wird, als dass dieses Wesen selber handeln könnte. Die Zentralfigur von Ubu Roi ist im Grunde eine Marionette, die andere Figuren wie Marionetten für sich agieren lässt.

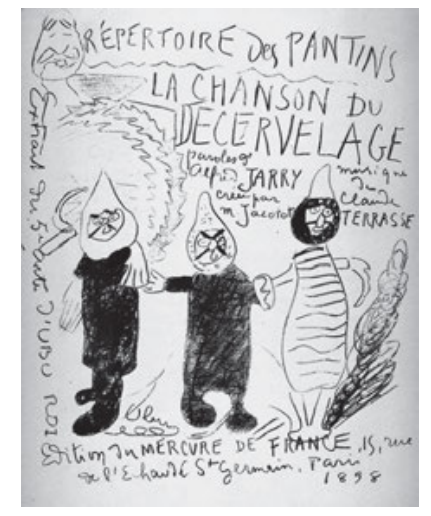

Abb. 43: Album-Cover La Chanson du décervelage. Zeichnung von Alfred Jarry.

Um Ihnen noch ein letztes Mal einen direkten Einblick in dieses prä-avantgardistische Spektakel zu geben, in die ganze Wucht dieses Schauspiels, seine Komik und Derbheit, möchte ich Ihnen eine weitere Passage aus Ubu Roi vorstellen, diesmal aus der ersten Szene des fünften und letzten Aktes. Es handelt sich um eine Abfolge von Auseinandersetzungen zunächst zwischen Vater und Mutter Ubu, die sich wie gewöhnlich wieder zusammenraufen, mit ihren Feinden, dem legitimen Thronanwärter und dessen polnischen Soldaten:

VATER UBU: Oh, aber jetzt doch, komm hierher, Du Aas! Auf die Knie mit Dir vor Deinem Herren (er stößt sie und wirft sie auf die Knie), Jetzt erwarte Deine letzten Qualen.

MUTTER UBU: Ho, Ho, Herr Ubu!

VATER UBU: Oh! Oh! Oh! Bist Du jetzt endlich fertig? Jetzt beginne ich: Verdrehung der Nase, Ausreißen der Haare, Hineinstechen des kleinen Holzstückchens in die Ohrlen, Extraktion des Gehirns durch die Fersen, Zerfetzen des Hinterns, teilweises oder totales Zerquetschen des Rückenmarks (wenn ihr das zumindest ihre charakterlichen Gräten zerstören würde), 
dabei Öffnung der Schwimmblase nicht zu vergessen und schließlich und endlich die wiederholte große Enthauptung von Johannes dem Täufer, dies alles herausgezogen aus der Heiligen Schrift, ebenso des Alten wie des Neuen Testaments, neu geordnet, verbessert und vervollkommnet durch den hier anwesenden Herren der Finanzen! Passt Dir das, Du Wurst? (Er zerreißt sie.)

MUTTER UBU: Gnade, Herr Ubu!

(Lauter Lärm am Eingang der Höhle.)

(Bougrelas, der sich mit seinen Soldaten in die Höhle stürzt.)

BOUGRELAS: Vorwärts, meine Freunde! Es lebe Polen!

VATER UBU: Oh! Oh! Warten Sie einen Augenblick, Herr Pololone. Warten Sie, bis ich mit Madame meiner besseren Hälfte fertig bin!

BOUGRELAS (schlägt ihn): Hier hast Du, Du Feigling, du Krücke, du Nichtsnutz, Du Häretiker, Du Muselmann!

VATER UBU (zurückschlagend): Hier nimm, Du Pololone, Du Trunkenbold, Du Bastard, Du Husar, Du Tartar, Du Kommisarde, Du Mansarde, Du Verpfeifarde, Du Savoyarde und Kommunarde!

MUTTER UBU: (sie schlägt ihn auch): Hier hast Du, Du Schrein, Du Schwein, Du Frollein, Du Hysterlein, Du Dieblein, Du Drecklein, Du Pololein! ${ }^{26}$

Welch ein Spektakel ist hier auf die Bühne zu bringen! Kein Wunder, dass derlei Kaskaden der Gewalt rasch auf die Zuschauerinnen und Zuschauer übersprangen. Die Sprachspiele, welche Alfred Jarry in derlei Momenten in seinen Text einführt, unterstützen lediglich das Theatralische, Szenische und Parodistische einer Handlung, die von Beginn an eigentlich an Dürftigkeit kaum zu überbieten war. Alles liegt hier in der Performance, liegt im Ausagieren von Trieben und Instinkten, die tief im Unbewussten des Menschen schlummern und auf diese Weise zumindest ans Licht kommen. Diese Prügelszenen mit ihren wüsten und zugleich kindlich-lyrischen Beschimpfungen erinnern an die Prügeleien auf dem Schulhof, ja an die Grausamkeiten von Kindern, die sich im Sandkasten mit der Schaufel auf den Kopf schlagen, bevor ihre Eltern einschreiten können. All dies ist auf der Bühne, und all dies ist vom Autor intendiert! Denn Vater Ubu und seine Frau sind nicht besser und nicht schlechter als wir alle: Sie repräsentieren den Menschen gerade in seiner uferlosen Unmenschlichkeit.

Die Wirkung dieses so ungeheuer verdichteten Stücks war fürwahr ungeheuerlich, hatte Alfred Jarry doch mit seinem Ubu-Zyklus ein Fresko des Bürgertums geschaffen, für das im Übrigen der Ausdruck „Kommunarde“ oder „,communard“, der auf die Pariser Kommune verweist, in der Tat zu einem Schimpfwort geworden war. Gerade auch die „communards“ hatte man gnadenlos gejagt und massakriert, um wieder Ordnung und Ruhe im Staate Frankreich herzustellen.

26 Jarry, Alfred: Ubu Roi, Acte V, Scène I/II, S. 83 ff. 
Die extreme Brutalität und Grausamkeit, die sich im Verlauf des Stücks immer wieder zeigt, sobald Vater und Mutter Ubu dazu Gelegenheit finden, wirkte insbesondere auf Antonin Artaud und Roger Vitrac, die das avantgardistische Theater in späteren Jahrzehnten mit ihren dramaturgischen Schöpfungen beleben sollten. Der erstgenannte übersetzte diese Problematik in die Ästhetik seines eigenen „Thêâtre de la cruaute“, mit dem wir uns im Verlauf unserer Vorlesung noch beschäftigen wollen. Nun aber gilt es, die weiteren Entwicklungen der Avantgarde nach den Futuristen zu untersuchen und uns dabei der Bewegung des Dadaismus zuzuwenden, mit welcher wir etwa bei unserer Beschäftigung mit Kurt Schwitters bereits Kontakt aufgenommen hatten. 